\title{
TEMPORAL CONVERGENCE OF A LOCALLY IMPLICIT DISCONTINUOUS GALERKIN METHOD FOR MAXWELL'S EQUATIONS *
}

\author{
Ludovic MOYA ${ }^{1}$
}

\begin{abstract}
In this paper we study the temporal convergence of a locally implicit discontinuous Galerkin method for the time-domain Maxwell's equations modeling electromagnetic waves propagation. Particularly, we wonder whether the method retains its second-order ordinary differential equation (ODE) convergence under stable simultaneous space-time grid refinement towards the true partial differential equation (PDE) solution. This is not a priori clear due to the component splitting which can introduce order reduction
\end{abstract}

Mathematics Subject Classification. 65M12, 65M60, 78M10.

Received June 8, 2011. Revised December 30, 2011.

Published online March 27, 2012.

\section{INTRODUCTION}

Nowadays, many different types of methods exist for the numerical resolution of time-domain Maxwell's equations modeling electromagnetic wave propagation. The most prominent method among physicists and engineers is still the finite difference time-domain (FDTD) method based on Yee's scheme [31]. This popularity is mainly due to its simplicity and efficiency in discretising simple domain problems. However, its inability to effectively handle complex geometries has prompted to search for alternatives methods. Also one of the main features of numerical methods based on finite element meshes like finite element time-domain (FETD) [17], finite volume time-domain (FVTD) [24] or discontinuous Galerkin time-domain (DGTD) $[5,10,14,15]$ methods is the possibility of using locally refined and non-conformal space grids to easily deal with complex geometries. In recent years there has been an increasing interest in the DGTD method. The latter is particularly well suited to the design of $h p$-adaptive strategies (i.e. where the characteristic mesh size $h$ and the interpolation degree $p$ change locally wherever it is needed) [8]. Thus the DGTD method can achieve a high order of accuracy and is used in many applications $[4,15]$.

In the same time the choice of the temporal integration method is a crucial step for the global efficiency of the numerical method. We distinguish two major families for the temporal integration: implicit and explicit methods. An implicit integration method to numerically solve a time-dependent PDE leads in general to unconditional

\footnotetext{
Keywords and phrases. Temporal convergence, discontinuous Galerkin method, time-domain Maxwell equations, component splitting, order reduction.

* In memory of Prof. dr. Jan Verwer, who passed away unexpectedly before completion of this paper on 16 February 2011, at his home in Heiloo.

1 INRIA Sophia Antipolis - Méditerannée, NACHOS project-team, 2004 Route des Lucioles, BP 93, 06902 Sophia Antipolis Cedex, France. ludovic.moya@inria.fr
} 
stability. Then the time step can be chosen arbitrarily large. However, implicit methods require the solution of large linear systems resulting in a high computational effort. An explicit integration method results in less computational effort per time step, but readily leads to unduly step size restrictions caused by the smallest grid elements. For examples of implicit and explicit integration methods for the semi-discrete Maxwell's equations we refer to $[3,29]$ and $[1,10]$, respectively. A possible alternative to overcome step size limitation, induced by local mesh refinements in explicit methods, is to use smaller time steps precisely where the smallest elements are located, given by a local stability criterion. These local time-stepping techniques have been recently studied for second-order wave equations discretized in space by continuous or discontinuous finite element methods in [6] and more specifically for the time-domain Maxwell's equations discretized in space by a discontinuous Galerkin method in [11, 20,26]. Considering the strengths of implicit and explicit methods, the authors of $[7,23,27]$ have proposed another alternative: locally implicit time integration methods. More precisely, the smallest grid elements are treated implicitly and the remaining elements explicitly by a technique we call component splitting. If the ratio of fine to coarse elements is small, the most severe step size restrictions are overcome. The counterpart of this approach is having to solve per time step a linear system. But due to the assumed small fine to coarse cell size ratio, the overhead will also be small while the solution can be advanced in time with step sizes determined by the coarse elements. Consequently, these methods are particularly well suited if the local refinement is strongly localized. Note that the method from $[7,23]$ has been especially designed for a discontinuous Galerkin discretization, while that from [27] covers the common spatial discretizations like finite-difference and various finite-element discretizations.

In this paper we study the temporal convergence of the locally implicit DGTD method for Maxwell's equations initially proposed by Piperno in [23]. In particular we examine whether the method retains its second-order ODE convergence towards the true PDE solution under stable simultaneous space-time grid refinement. This question is legitimate because component splitting can cause order reduction.

The paper is organized as follows. Section 2 presents the problem and the notations. In Section 3 we introduce the component splitting and the implicit-explicit method for time-domain Maxwell's equations spatially discretized with a discontinuous Galerkin method. This implicit-explicit integration method is a blend of the explicit one-step second-order Leap-Frog scheme (LF2) and the implicit one-step second-order Crank-Nicolson scheme (CN2). Next we study the temporal convergence order. The different steps of this convergence analysis are the same as in [27] where the author proposes another implicit-explicit integration method for the semidiscrete Maxwell's equations. The latter method is also a blend of LF2 and CN2 and retains its second-order ODE convergence towards the true PDE solution under stable simultaneous space-time grid refinement [27]. In Section 4 we give some numerical results for 2D Maxwell problems to illustrate the previous convergence analysis. Section 5 concludes the paper with final remarks and future plans.

\section{Problem Statement}

We consider time-domain Maxwell's equations

$$
\left\{\begin{array}{l}
\epsilon \partial_{t} E=\operatorname{curl} H-\sigma E-J_{E}, \\
\mu \partial_{t} H=-\operatorname{curl} E,
\end{array}\right.
$$

where $E$ and $H$ denote the electric and magnetic field, respectively. $J_{E}$ is the given source current and $\epsilon, \mu$ and $\sigma$ are coefficients representing dielectric permittivity, magnetic permeability and conductivity, respectively. After discretization in space by a DG method we obtain the semi-discrete Maxwell system

$$
\left\{\begin{array}{l}
M^{\epsilon} \partial_{t} E=S H-D E+M^{\epsilon} f^{E}, \\
M^{\mu} \partial_{t} H=-S^{T} E+M^{\mu} f^{H},
\end{array}\right.
$$

where, for convenience, we use the same notation for the electric and magnetic fields $E$ and $H$ as in the spacecontinuous case. For more details on DG spatial discretization we refer to [7]. The matrices $M^{\epsilon}, M^{\mu}$ are the DG 
mass matrices which contain the values of the dielectric permittivity and magnetic permeability coefficient. The matrix $S$ emanates from the discretization of the curl operator. The matrix $D$ is associated with the dissipative conduction term $-\sigma E$. Throughout $D$ may be assumed symmetric positive semi-definite. The functions $f^{E}$ and $f^{H}$ are associated with source terms. More precisely $f^{E}$ represents the given source current $-J_{E}$, but $f^{E}$ and $f^{H}$ may also contain Dirichlet boundary data.

We can give an equivalent formulation of (2.2) without mass matrix. As in [1] we introduce the Cholesky factorizations

$$
M^{\epsilon}=L_{M^{\epsilon}} L_{M^{\epsilon}}^{T} \text { and } M^{\mu}=L_{M^{\mu}} L_{M^{\mu}}^{T},
$$

where $L_{M^{\epsilon}}$ and $L_{M^{\mu}}$ are triangular matrices. Then with (2.2) we have

$$
\left\{\begin{array}{l}
L_{M^{\epsilon}} L_{M^{\epsilon}}^{T} \partial_{t} E=S H-D E+L_{M^{\epsilon}} L_{M^{\epsilon}}^{T} f^{E} \\
L_{M^{\mu}} L_{M^{\mu}}^{T} \partial_{t} H=-S^{T} E+L_{M^{\mu}} L_{M^{\mu}}^{T} f^{H}
\end{array}\right.
$$

Introducing $\tilde{E}=L_{M^{\epsilon}}^{T} E$ and $\tilde{H}=L_{M^{\mu}}^{T} H$, we get

$$
\left\{\begin{array}{l}
\partial_{t} \tilde{E}=L_{M^{\epsilon}}^{-1} S\left(L_{M^{\mu}}^{T}\right)^{-1} \tilde{H}-L_{M^{\epsilon}}^{-1} D\left(L_{M^{\epsilon}}^{T}\right)^{-1} \tilde{E}+L_{M^{\epsilon}}^{T} f^{E} \\
\partial_{t} \tilde{H}=-L_{M^{\mu}}^{-1} S^{T}\left(L_{M^{\epsilon}}^{T}\right)^{-1} \tilde{E}+L_{M^{\mu}}^{T} f^{H}
\end{array}\right.
$$

Next we write

$$
\begin{aligned}
& \tilde{S}=L_{M^{\epsilon}}^{-1} S\left(L_{M^{\mu}}^{-1}\right)^{T}, \quad \tilde{D}=L_{M^{\epsilon}}^{-1} D\left(L_{M^{\epsilon}}^{-1}\right)^{T}, \\
& \tilde{f}^{E}=L_{M^{\epsilon}}^{T} f^{E}, \quad \tilde{f}^{H}=L_{M^{\mu}}^{T} f^{H},
\end{aligned}
$$

and note that

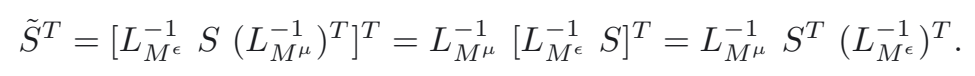

Thus we can write the semi-discrete Maxwell system equivalent to (2.2) as

$$
\left\{\begin{array}{l}
\partial_{t} \tilde{E}=\tilde{S} \tilde{H}-\tilde{D} \tilde{E}+\tilde{f}^{E} \\
\partial_{t} \tilde{H}=-\tilde{S}^{T} \tilde{E}+\tilde{f}^{H}
\end{array}\right.
$$

For convenience of notation and presentation we use the same notation in (2.2) and (2.8) i.e.

$$
\left\{\begin{array}{l}
\partial_{t} E=S H-D E+f^{E} \\
\partial_{t} H=-S^{T} E+f^{H}
\end{array}\right.
$$

We will proceed with (2.9), the meaning of $E, H, S, D, f^{E}$ and $f^{H}$ will always be clear from the context or will be precised. In particular results obtained for (2.9) apply to (2.2) and vice versa. Note that $S$ emanates from an appropriate DG discretization for the Maxwell problem under consideration and further on we will prove that

$$
S \sim \frac{1}{h}, \quad \text { for } h \rightarrow 0
$$

where the parameter $h$ denotes the maximum diameter of the (non-uniform) grid elements. Throughout the remainder we will assume initial values at time $t=0$ and the source functions $f^{E}(t), f^{H}(t) \in \mathrm{C}^{2}[0, T]$ for an interval $[0, T]$, so that $E(t), H(t) \in \mathrm{C}^{3}[0, T]$. 


\section{The implicit-explicit DGTD METHOD}

The implicit-explicit integration considered in this note is issued from $[7,23]$. As we have previously mentioned it is a blend of the second order LF2 scheme that we write in the three-stage form, emanating from Verlet's method, see [23]

$$
\left\{\begin{array}{l}
\frac{H^{n+\frac{1}{2}}-H^{n}}{\Delta t / 2}=-S^{T} E^{n}+f^{H}\left(t_{n}\right), \\
\frac{E^{n+1}-E^{n}}{\Delta t}=S H^{n+\frac{1}{2}}-\frac{1}{2} D\left(E^{n+1}+E^{n}\right)+\frac{1}{2}\left(f^{E}\left(t_{n+1}\right)+f^{E}\left(t_{n}\right)\right), \\
\frac{H^{n+1}-H^{n+\frac{1}{2}}}{\Delta t / 2}=-S^{T} E^{n+1}+f^{H}\left(t_{n+1}\right),
\end{array}\right.
$$

and the second order, unconditionally stable CN2 scheme that we also write in the three-stage form

$$
\left\{\begin{array}{l}
\frac{H^{n+\frac{1}{2}}-H^{n}}{\Delta t / 2}=-S^{T} E^{n}+f^{H}\left(t_{n}\right), \\
\frac{E^{n+1}-E^{n}}{\Delta t}=S H^{n+1}-\frac{1}{2} D\left(E^{n+1}+E^{n}\right)+\frac{1}{2}\left(f^{E}\left(t_{n+1}\right)+f^{E}\left(t_{n}\right)\right), \\
\frac{H^{n+1}-H^{n+\frac{1}{2}}}{\Delta t / 2}=-S^{T} E^{n+1}+f^{H}\left(t_{n+1}\right) .
\end{array}\right.
$$

which only differ in the middle stage in the time level for $H$ (see [27]). Herein $\Delta t=t_{n+1}-t_{n}$ denotes the step size and upper indices time levels, as usual.

\subsection{Component splitting}

The set of grid elements is assumed to be partitioned into two subsets: one made of the smallest elements that will be treated implicitly using the CN2 method and the other one of the remaining elements that will be treated explicitly with the LF2 method. In line with this splitting the problem unknowns are reordered as

$$
E=\left(\begin{array}{c}
E_{e} \\
E_{i}
\end{array}\right) \quad \text { and } H=\left(\begin{array}{c}
H_{e} \\
H_{i}
\end{array}\right),
$$

where the indices $i$ and $e$ are associated to the elements of the subset treated implicitly and explicitly, respectively. Likewise the semi-discrete curl operator $S$ is split into the block form

$$
S=\left(\begin{array}{cc}
S_{e} & -A_{e i} \\
-A_{i e} & S_{i}
\end{array}\right),
$$

for the specific meaning of the block-entries of $S$ we refer to [7]. $D$ is supposed to be split accordingly into

$$
D=\left(\begin{array}{cc}
D_{e} & 0 \\
0 & D_{i}
\end{array}\right)
$$

and we write

$$
f^{E}=\left(\begin{array}{c}
f_{e}^{E} \\
f_{i}^{E}
\end{array}\right), \quad f^{H}=\left(\begin{array}{c}
f_{e}^{H} \\
f_{i}^{H}
\end{array}\right) .
$$


Inserting this splitting into (2.9) we obtain the system of ODEs

$$
\left\{\begin{array}{l}
\partial_{t} E_{e}=S_{e} H_{e}-A_{e i} H_{i}-D_{e} E_{e}+f_{e}^{E}(t), \\
\partial_{t} E_{i}=S_{i} H_{i}-A_{i e} H_{e}-D_{i} E_{i}+f_{i}^{E}(t), \\
\partial_{t} H_{e}=-S_{e}^{T} E_{e}+A_{i e}^{T} E_{i}+f_{e}^{H}(t), \\
\partial_{t} H_{i}=-S_{i}^{T} E_{i}+A_{e i}^{T} E_{e}+f_{i}^{H}(t) .
\end{array}\right.
$$

\subsection{The implicit-explicit time integration method}

The implicit-explicit method proposed in $[7,23]$ is a blend of LF2 and CN2 applied to (3.7). It reads

$$
\begin{aligned}
& \left\{\begin{array}{l}
\frac{H_{e}^{n+1 / 2}-H_{e}^{n}}{\Delta t / 2}=-S_{e}^{T} E_{e}^{n}+A_{i e}^{T} E_{i}^{n}+f_{e}^{H}\left(t_{n}\right), \\
\frac{E_{e}^{n+1 / 2}-E_{e}^{n}}{\Delta t / 2}=S_{e} H_{e}^{n+1 / 2}-A_{e i} H_{i}^{n}-D_{e} E_{e}^{n}+f_{e}^{E}\left(t_{n}\right),
\end{array}\right. \\
& \left\{\begin{aligned}
\frac{E_{i}^{n+1}-E_{i}^{n}}{\Delta t}= & S_{i}\left(\frac{H_{i}^{n+1}+H_{i}^{n}}{2}\right)-A_{i e} H_{e}^{n+1 / 2} \\
& -D_{i}\left(\frac{E_{i}^{n+1}+E_{i}^{n}}{2}\right)+\frac{f_{i}^{E}\left(t_{n+1}\right)+f_{i}^{E}\left(t_{n}\right)}{2}, \\
\frac{H_{i}^{n+1}-H_{i}^{n}}{\Delta t}= & -S_{i}^{T}\left(\frac{E_{i}^{n+1}+E_{i}^{n}}{2}\right)+A_{e i}^{T} E_{e}^{n+1 / 2}+\frac{f_{i}^{H}\left(t_{n+1}\right)+f_{i}^{H}\left(t_{n}\right)}{2},
\end{aligned}\right. \\
& \left\{\begin{array}{l}
\frac{E_{e}^{n+1}-E_{e}^{n+1 / 2}}{\Delta t / 2}=S_{e} H_{e}^{n+1 / 2}-A_{e i} H_{i}^{n+1}-D_{e} E_{e}^{n+1}+f_{e}^{E}\left(t_{n+1}\right), \\
\frac{H_{e}^{n+1}-H_{e}^{n+1 / 2}}{\Delta t / 2}=-S_{e}^{T} E_{e}^{n+1}+A_{i e}^{T} E_{i}^{n+1}+f_{e}^{H}\left(t_{n+1}\right) .
\end{array}\right.
\end{aligned}
$$

For the stability analysis of this method we refer to [7]. The proof is based on the conservation of a discrete electromagnetic energy. It is proved that this energy is a positive quadratic form of the numerical unknowns $E_{e}^{n}, E_{i}^{n}, H_{e}^{n}$ and $H_{i}^{n}$ under a condition on the time step size. Consequently the non-dissipative nature of the method yields to the stability of the method.

\subsection{Matrix behavior for $h \rightarrow 0$}

In this subsection we are interested in the behavior of the matrices in (3.8) for $h \rightarrow 0$. This is an essential point for convergence analysis because some of these matrices can lie at the origin of order reduction. Let us consider the general case of dimension $d(d=1,2$ or 3$)$. First we investigate the behavior of the matrices in the formulation with the mass matrices. Thereafter we will be able to deduce the behavior of the matrices in (3.8).

We reintroduce the notation with a tilde for the elements involved in the formulation without mass matrix (see (2.8)) in order to avoid confusion. The specific meaning of the block-entries of the different matrices involved in the formulation with mass matrices can be found in [7]. First we observe that the mass matrices are only composed of volumic terms, hence we have

$$
M^{\epsilon}, M^{\mu} \backsim h^{d}, \text { for } h \rightarrow 0 .
$$

Thus

$$
L_{M^{\epsilon}}, L_{M^{\epsilon}}^{T}, L_{M^{\mu}} \text { and } L_{M^{\mu}}^{T} \backsim h^{\frac{d}{2}} \text {, for } h \rightarrow 0 \text {. }
$$


The matrices $S_{e}$ and $S_{i}$ are composed of volumic and surfacic terms, hence

$$
S_{e} \text { and } S_{i} \backsim h^{d-1} \text {, for } h \rightarrow 0 .
$$

The matrices $A_{e i}$ and $A_{i e}$ represent surfacic terms (interface matrices). Hence

$$
A_{e i} \text { and } A_{i e} \backsim h^{d-1} \text {, for } h \rightarrow 0
$$

and from the block form of the matrix $S$ (see (3.4)) we deduce with (3.11) and (3.12) that

$$
S \backsim h^{d-1} \text {, for } h \rightarrow 0 .
$$

From (2.6) we get

$$
\tilde{S}=L_{M^{\epsilon}}^{-1} S\left(L_{M^{\mu}}^{-1}\right)^{T},
$$

then with the behaviors above we deduce that for $h \rightarrow 0$

$$
\begin{aligned}
& \tilde{S}_{e}, \tilde{S}_{i}=\mathcal{O}\left(\frac{1}{h}\right), \\
& \tilde{A}_{e i}, \tilde{A}_{i e}=\mathcal{O}\left(\frac{1}{h}\right),
\end{aligned}
$$

and we have the expected behavior $(2.10)$ for $\tilde{S}$.

\subsection{Temporal convergence}

In this section we are interested in the PDE convergence of method (3.8). More precisely, we will examine whether the method retains its second-order ODE convergence under stable simultaneous space-time grid refinement $\Delta t \backsim h, h \rightarrow 0$ towards the true PDE solution. This is not a priori clear due to the component splitting which can introduce order reduction through error constants which grow with $h^{-1}$, for $h \rightarrow 0$.

This section is organized in four subsections. In Section 3.4.1 we will eliminate the intermediate values of (3.8) to get an equivalent one step formula from $t_{n}$ to $t_{n+1}$ that we will use for our convergence analysis. In Section 3.4.2 we will introduce the perturbed method obtained by substituting the true PDE solution restricted to the assumed space grid into (3.7), and defects (space-time truncation errors) obtained by substituting this true PDE solution into the equivalent one step formula of our method. In Section 3.4.3 we will define the common one-step recurrence relation for the global error. In Section 3.4.4 we will point out the order reduction mentioned above. Finally in Section 3.4.5 we will see that this order reduction, affecting the local error, may (partly) cancel in the transition from the local to the global error.

\subsubsection{Elimination of intermediates values}

First we treat $H_{e}$. From the first and last equations of (3.8) we get

$$
\begin{aligned}
& H_{e}^{n+\frac{1}{2}}=H_{e}^{n}-\frac{\Delta t}{2} S_{e}^{T} E_{e}^{n}+\frac{\Delta t}{2} A_{i e}^{T} E_{i}^{n}+\frac{\Delta t}{2} f_{e}^{H}\left(t_{n}\right), \\
& H_{e}^{n+\frac{1}{2}}=H_{e}^{n+1}+\frac{\Delta t}{2} S_{e}^{T} E_{e}^{n+1}-\frac{\Delta t}{2} A_{i e}^{T} E_{i}^{n+1}+\frac{\Delta t}{2} f_{e}^{H}\left(t_{n+1}\right) .
\end{aligned}
$$

Inserting the first equation of (3.16) into the last equation of (3.8) yields

$$
H_{e}^{n+1}=H_{e}^{n}-\frac{\Delta t}{2} S_{e}^{T}\left(E_{e}^{n}+E_{e}^{n+1}\right)+\frac{\Delta t}{2} A_{i e}^{T}\left(E_{i}^{n}+E_{i}^{n+1}\right)+\frac{\Delta t}{2}\left(f_{e}^{H}\left(t_{n}\right)+f_{e}^{H}\left(t_{n+1}\right)\right) .
$$


Next we treat $E_{e}$. From the second and fifth equations of (3.8) we get

$$
\begin{aligned}
& E_{e}^{n+\frac{1}{2}}=E_{e}^{n}+\frac{\Delta t}{2} S_{e} H_{e}^{n+\frac{1}{2}}-\frac{\Delta t}{2} A_{e i} H_{i}^{n}-\frac{\Delta t}{2} D_{e} E_{e}^{n}+\frac{\Delta t}{2} f_{e}^{E}\left(t_{n}\right), \\
& E_{e}^{n+\frac{1}{2}}=E_{e}^{n+1}-\frac{\Delta t}{2} S_{e} H_{e}^{n+\frac{1}{2}}+\frac{\Delta t}{2} A_{e i} H_{i}^{n+1}+\frac{\Delta t}{2} D_{e} E_{e}^{n+1}-\frac{\Delta t}{2} f_{e}^{E}\left(t_{n+1}\right) .
\end{aligned}
$$

Inserting the first equation of (3.18) and half of each expression of (3.16) for $H_{e}^{n+\frac{1}{2}}$ into the fifth equation of (3.8) yields

$$
\begin{aligned}
E_{e}^{n+1}= & E_{e}^{n}+\frac{\Delta t}{2} S_{e}\left(H_{e}^{n}+H_{e}^{n+1}\right)-\frac{\Delta t}{2} A_{e i}\left(H_{i}^{n}+H_{i}^{n+1}\right)-\frac{\Delta t}{2} D_{e}\left(E_{e}^{n}+E_{e}^{n+1}\right)+\frac{\Delta t}{2}\left(f_{e}^{E}\left(t_{n}\right)+f_{e}^{E}\left(t_{n+1}\right)\right) \\
& +\frac{\Delta t^{2}}{4} S_{e}\left(-S_{e}^{T} E_{e}^{n}+A_{i e}^{T} E_{i}^{n}\right)+\frac{\Delta t^{2}}{4} S_{e}\left(S_{e}^{T} E_{e}^{n+1}-A_{i e}^{T} E_{i}^{n+1}\right)+\frac{\Delta t^{2}}{4} S_{e}\left(f_{e}^{H}\left(t_{n}\right)+f_{e}^{H}\left(t_{n+1}\right)\right) .
\end{aligned}
$$

Now we consider $H_{i}$. Inserting half of each expression of (3.18) for $E_{e}^{n+\frac{1}{2}}$ in the fourth equation of (3.8) gives

$$
\begin{aligned}
H_{i}^{n+1}= & H_{i}^{n}-\frac{\Delta t}{2} S_{i}^{T}\left(E_{i}^{n}+E_{i}^{n+1}\right)+\frac{\Delta t}{2} A_{e i}^{T}\left(E_{e}^{n}+E_{e}^{n+1}\right)+\frac{\Delta t}{2}\left(f_{i}^{H}\left(t_{n}\right)+f_{i}^{H}\left(t_{n+1}\right)\right) \\
& +\frac{\Delta t^{2}}{4} A_{e i}^{T} A_{e i}\left(H_{i}^{n+1}-H_{i}^{n}\right)+\frac{\Delta t^{2}}{4} A_{e i}^{T} D_{e}\left(E_{e}^{n+1}-E_{e}^{n}\right)+\frac{\Delta t^{2}}{4} A_{e i}^{T}\left(f_{e}^{E}\left(t_{n}\right)+f_{e}^{E}\left(t_{n+1}\right)\right) .
\end{aligned}
$$

Finally we treat $E_{i}$. Inserting half of each expression of (3.16) for $H_{e}^{n+\frac{1}{2}}$ in the third equation of (3.8) yields

$$
\begin{aligned}
E_{i}^{n+1}= & E_{i}^{n}+\frac{\Delta t}{2} S_{i}\left(H_{i}^{n}+H_{i}^{n+1}\right)-\frac{\Delta t}{2} A_{i e}\left(H_{e}^{n}+H_{e}^{n+1}\right)-\frac{\Delta t}{2} D_{i}\left(E_{i}^{n}+E_{i}^{n+1}\right)+\frac{\Delta t}{2}\left(f_{i}^{E}\left(t_{n}\right)+f_{i}^{E}\left(t_{n+1}\right)\right) \\
& -\frac{\Delta t^{2}}{4} A_{i e}\left(-S_{e}^{T} E_{e}^{n}+A_{i e}^{T} E_{i}^{n}\right)-\frac{\Delta t^{2}}{4} A_{i e}\left(S_{e}^{T} E_{e}^{n+1}-A_{i e}^{T} E_{i}^{n+1}\right)-\frac{\Delta t^{2}}{4} A_{i e}\left(f_{e}^{H}\left(t_{n}\right)+f_{e}^{H}\left(t_{n+1}\right)\right) .
\end{aligned}
$$

The equivalent method of (3.8) with its intermediate values eliminated thus reads

$$
\begin{aligned}
E_{e}^{n+1}= & E_{e}^{n}+\frac{\Delta t}{2} S_{e}\left(H_{e}^{n}+H_{e}^{n+1}\right)-\frac{\Delta t}{2} A_{e i}\left(H_{i}^{n}+H_{i}^{n+1}\right)-\frac{\Delta t}{2} D_{e}\left(E_{e}^{n}+E_{e}^{n+1}\right)+\frac{\Delta t}{2}\left(f_{e}^{E}\left(t_{n}\right)+f_{e}^{E}\left(t_{n+1}\right)\right) \\
& +\frac{\Delta t^{2}}{4} S_{e}\left[\left(-S_{e}^{T} E_{e}^{n}+A_{i e}^{T} E_{i}^{n}\right)-\left(S_{e}^{T} E_{e}^{n+1}-A_{i e}^{T} E_{i}^{n+1}\right)\right]+\frac{\Delta t^{2}}{4} S_{e}\left(f_{e}^{H}\left(t_{n}\right)+f_{e}^{H}\left(t_{n+1}\right)\right), \\
E_{i}^{n+1}= & E_{i}^{n}+\frac{\Delta t}{2} S_{i}\left(H_{i}^{n}+H_{i}^{n+1}\right)-\frac{\Delta t}{2} A_{i e}\left(H_{e}^{n}+H_{e}^{n+1}\right)-\frac{\Delta t}{2} D_{i}\left(E_{i}^{n}+E_{i}^{n+1}\right)+\frac{\Delta t}{2}\left(f_{i}^{E}\left(t_{n}\right)+f_{i}^{E}\left(t_{n+1}\right)\right) \\
& -\frac{\Delta t^{2}}{4} A_{i e}\left[\left(-S_{e}^{T} E_{e}^{n}+A_{i e}^{T} E_{i}^{n}\right)-\left(S_{e}^{T} E_{e}^{n+1}-A_{i e}^{T} E_{i}^{n+1}\right)\right]-\frac{\Delta t^{2}}{4} A_{i e}\left(f_{e}^{H}\left(t_{n}\right)+f_{e}^{H}\left(t_{n+1}\right)\right), \\
H_{e}^{n+1}= & H_{e}^{n}-\frac{\Delta t}{2} S_{e}^{T}\left(E_{e}^{n}+E_{e}^{n+1}\right)+\frac{\Delta t}{2} A_{i e}^{T}\left(E_{i}^{n}+E_{i}^{n+1}\right)+\frac{\Delta t}{2}\left(f_{e}^{H}\left(t_{n}\right)+f_{e}^{H}\left(t_{n+1}\right)\right), \\
H_{i}^{n+1}= & H_{i}^{n}-\frac{\Delta t}{2} S_{i}^{T}\left(E_{i}^{n}+E_{i}^{n+1}\right)+\frac{\Delta t}{2} A_{e i}^{T}\left(E_{e}^{n}+E_{e}^{n+1}\right)+\frac{\Delta t}{2}\left(f_{i}^{H}\left(t_{n}\right)+f_{i}^{H}\left(t_{n+1}\right)\right) \\
& +\frac{\Delta t^{2}}{4} A_{e i}^{T} A_{e i}\left(H_{i}^{n+1}-H_{i}^{n}\right)+\frac{\Delta t^{2}}{4} A_{e i}^{T}\left[D_{e}\left(E_{e}^{n+1}-E_{e}^{n}\right)+\left(f_{e}^{E}\left(t_{n}\right)+f_{e}^{E}\left(t_{n+1}\right)\right)\right] .
\end{aligned}
$$




\subsubsection{The perturbed method and defects for the PDE solution}

Let $E_{e}^{h}(t)$ denote at time $t$ the true solution of the PDE problem restricted to the assumed space grid that we have approximated with the semi-discrete system $(3.7) . E_{e}^{h}\left(t_{n}\right)$ thus represents the vector that is approximated by $E_{e}^{n}$. Assume the same notation for $E_{i}, H_{e}$ and $H_{i}$. Substituting $E_{e}^{h}(t), E_{i}^{h}(t), H_{e}^{h}(t)$ and $H_{i}^{h}(t)$ into (3.7) reveals the spatial truncation errors

$$
\left\{\begin{array}{l}
\frac{\mathrm{d}}{\mathrm{d} t} E_{e}^{h}(t)=S_{e} H_{e}^{h}(t)-A_{e i} H_{i}^{h}(t)-D_{e} E_{e}^{h}(t)+f_{e}^{E}(t)+\sigma_{e}^{E}(t), \\
\frac{\mathrm{d}}{\mathrm{d} t} E_{i}^{h}(t)=S_{i} H_{i}^{h}(t)-A_{i e} H_{e}^{h}(t)-D_{i} E_{i}^{h}(t)+f_{i}^{E}(t)+\sigma_{i}^{E}(t), \\
\frac{\mathrm{d}}{\mathrm{d} t} H_{e}^{h}(t)=-S_{e}^{T} E_{e}^{h}(t)+A_{i e}^{T} E_{i}^{h}(t)+f_{e}^{H}(t)+\sigma_{e}^{H}(t), \\
\frac{\mathrm{d}}{\mathrm{d} t} H_{i}^{h}(t)=-S_{i}^{T} E_{i}^{h}+A_{e i}^{T} E_{e}^{h}(t)+f_{i}^{H}(t)+\sigma_{i}^{H}(t),
\end{array}\right.
$$

where $\sigma_{e}^{E}(t), \sigma_{i}^{E}(t), \sigma_{e}^{H}(t)$ and $\sigma_{i}^{H}(t)$ denote the spatial truncation errors.

Substituting $E_{e}^{h}(t), E_{i}^{h}(t), H_{e}^{h}(t)$ and $H_{i}^{h}(t)$ into (3.22) reveals the defects for the PDE solution (space-time truncation errors) and gives what we call the perturbed method

$$
\begin{aligned}
E_{e}^{h}\left(t_{n+1}\right)= & E_{e}^{h}\left(t_{n}\right)+\frac{\Delta t}{2} S_{e}\left(H_{e}^{h}\left(t_{n}\right)+H_{e}^{h}\left(t_{n+1}\right)\right)-\frac{\Delta t}{2} A_{e i}\left(H_{i}^{h}\left(t_{n}\right)+H_{i}^{h}\left(t_{n+1}\right)\right) \\
& -\frac{\Delta t}{2} D_{e}\left(E_{e}^{h}\left(t_{n}\right)+E_{e}^{h}\left(t_{n+1}\right)\right)+\frac{\Delta t}{2}\left(f_{e}^{E}\left(t_{n}\right)+f_{e}^{E}\left(t_{n+1}\right)\right) \\
& +\frac{\Delta t^{2}}{4} S_{e}\left[\left(-S_{e}^{T} E_{e}^{h}\left(t_{n}\right)+A_{i e}^{T} E_{i}^{h}\left(t_{n}\right)\right)-\left(S_{e}^{T} E_{e}^{h}\left(t_{n+1}\right)-A_{i e}^{T} E_{i}^{h}\left(t_{n+1}\right)\right)\right] \\
& +\frac{\Delta t^{2}}{4} S_{e}\left(f_{e}^{H}\left(t_{n}\right)+f_{e}^{H}\left(t_{n+1}\right)\right)+\Delta t \delta_{e, n}^{E}, \\
E_{i}^{h}\left(t_{n+1}\right)= & E_{i}^{h}\left(t_{n}\right)+\frac{\Delta t}{2} S_{i}\left(H_{i}^{h}\left(t_{n}\right)+H_{i}^{h}\left(t_{n+1}\right)\right)-\frac{\Delta t}{2} A_{i e}\left(H_{e}^{h}\left(t_{n}\right)+H_{e}^{h}\left(t_{n+1}\right)\right) \\
& -\frac{\Delta t}{2} D_{i}\left(E_{i}^{h}\left(t_{n}\right)+E_{i}^{h}\left(t_{n+1}\right)\right)+\frac{\Delta t}{2}\left(f_{i}^{E}\left(t_{n}\right)+f_{i}^{E}\left(t_{n+1}\right)\right) \\
& -\frac{\Delta t^{2}}{4} A_{i e}\left[\left(-S_{e}^{T} E_{e}^{h}\left(t_{n}\right)+A_{i e}^{T} E_{i}^{h}\left(t_{n}\right)\right)-\left(S_{e}^{T} E_{e}^{h}\left(t_{n+1}\right)-A_{i e}^{T} E_{i}^{h}\left(t_{n+1}\right)\right)\right] \\
& -\frac{\Delta t^{2}}{4} A_{i e}\left(f_{e}^{H}\left(t_{n}\right)+f_{e}^{H}\left(t_{n+1}\right)\right)+\Delta t \delta_{i, n}^{E}, \\
H_{e}^{h}\left(t_{n+1}\right)= & H_{e}^{h}\left(t_{n}\right)-\frac{\Delta t}{2} S_{e}^{T}\left(E_{e}^{h}\left(t_{n}\right)+E_{e}^{h}\left(t_{n+1}\right)\right)+\frac{\Delta t}{2} A_{i e}^{T}\left(E_{i}^{h}\left(t_{n}\right)+E_{i}^{h}\left(t_{n+1}\right)\right) \\
& +\frac{\Delta t}{2}\left(f_{e}^{H}\left(t_{n}\right)+f_{e}^{H}\left(t_{n+1}\right)\right)+\Delta t \delta_{e, n}^{H}, \\
& +\frac{\Delta t_{i}^{h}}{4} A_{e i}^{T}\left[t_{n}\right)-\frac{\Delta t}{2} S_{i}^{T}\left(E_{i}^{h}\left(t_{n}\right)+E_{i}^{h}\left(t_{n+1}\right)\right)+\frac{\Delta t}{2} A_{e i}^{T}\left(E_{e}^{h}\left(t_{n}\right)+E_{e}^{h}\left(t_{n+1}\right)\right)+\frac{\Delta t}{2}\left(f_{i}^{H}\left(t_{n}\right)+f_{i}^{H}\left(t_{n+1}\right)\right) \\
& +\frac{\Delta t^{2}}{4} A_{e i}^{T} A_{e i}\left(H_{i}^{h}\left(t_{n+1}\right)-H_{i}^{h}\left(t_{n}\right)\right) \\
H_{i}^{h}\left(t_{n+1}\right) & \left.\left.\left(t_{n}\right)\right)+\left(f_{e}^{E}\left(t_{n}\right)+f_{e}^{E}\left(t_{n+1}\right)\right)\right]+\Delta t \delta_{i, n}^{H}, \\
&
\end{aligned}
$$

where $\delta_{e, n}^{E}, \delta_{i, n}^{E}, \delta_{e, n}^{H}$ and $\delta_{i, n}^{H}$ denote the defects for the PDE solution. 
Eliminating all source term contributions $f_{e}^{E}, f_{i}^{E}, f_{e}^{H}$ and $f_{i}^{H}(3.23)$ yields

$$
\begin{aligned}
\delta_{e, n}^{E}= & \delta_{E_{e}^{h}}+\frac{\Delta t}{4} S_{e} \frac{\mathrm{d}}{\mathrm{d} t}\left(H_{e}^{h}\left(t_{n+1}\right)-H_{e}^{h}\left(t_{n}\right)\right)+\frac{1}{2}\left(\sigma_{e}^{E}\left(t_{n}\right)+\sigma_{e}^{E}\left(t_{n+1}\right)\right)+\frac{\Delta t}{4} S_{e}\left(\sigma_{e}^{H}\left(t_{n}\right)+\sigma_{e}^{H}\left(t_{n+1}\right)\right), \\
\delta_{i, n}^{E}= & \delta_{E_{i}^{h}}-\frac{\Delta t}{4} A_{i e} \frac{\mathrm{d}}{\mathrm{d} t}\left(H_{e}^{h}\left(t_{n+1}\right)-H_{e}^{h}\left(t_{n}\right)\right)+\frac{1}{2}\left(\sigma_{i}^{E}\left(t_{n}\right)+\sigma_{i}^{E}\left(t_{n+1}\right)\right)-\frac{\Delta t}{4} A_{i e}\left(\sigma_{e}^{H}\left(t_{n}\right)+\sigma_{e}^{H}\left(t_{n+1}\right)\right), \\
\delta_{e, n}^{H}= & \delta_{H_{e}^{h}}+\frac{1}{2}\left(\sigma_{e}^{H}\left(t_{n}\right)+\sigma_{e}^{H}\left(t_{n+1}\right)\right) \\
\delta_{i, n}^{H}= & \delta_{H_{i}^{h}}-\frac{\Delta t}{4} A_{e i}^{T} S_{e}\left(H_{e}^{h}\left(t_{n+1}\right)-H_{e}^{h}\left(t_{n}\right)\right)+\frac{\Delta t}{4} A_{e i}^{T} \frac{\mathrm{d}}{\mathrm{d} t}\left(E_{e}^{h}\left(t_{n+1}\right)-E_{e}^{h}\left(t_{n}\right)\right) \\
& +\frac{1}{2}\left(\sigma_{i}^{H}\left(t_{n}\right)+\sigma_{i}^{H}\left(t_{n+1}\right)\right)+\frac{\Delta t}{4} A_{e i}^{T}\left(\sigma_{e}^{E}\left(t_{n}\right)+\sigma_{e}^{E}\left(t_{n+1}\right)\right),
\end{aligned}
$$

where $\delta_{E_{e}^{h}}$ denotes the implicit trapezoidal $(\mathrm{CN})$ rule defect (see [27]) for variable $E_{e}^{h}$ (similarly for $E_{i}^{h}, H_{e}^{h}$ and $\left.H_{i}^{h}\right)$, i.e.

$$
\delta_{E_{e}^{h}}(t)=\frac{E_{e}^{h}(t+\Delta t)-E_{e}^{h}(t)}{\Delta t}-\frac{1}{2} \frac{\mathrm{d}}{\mathrm{d} t}\left(E_{e}^{h}(t+\Delta t)+E_{e}^{h}(t)\right) .
$$

\subsubsection{The error scheme}

Let $\varepsilon_{e, n}^{E}=E_{e}^{h}\left(t_{n}\right)-E_{e}^{n}$ denote the global error (similarly we introduce $\varepsilon_{i, n}^{E}, \varepsilon_{e, n}^{H}$ and $\left.\varepsilon_{i, n}^{H}\right)$. Substracting (3.22) and (3.24) we obtain the error scheme

$$
\begin{aligned}
\varepsilon_{e, n+1}^{E}= & \varepsilon_{e, n}^{E}+\frac{\Delta t}{2} S_{e}\left(\varepsilon_{e, n}^{H}+\varepsilon_{e, n+1}^{H}\right)-\frac{\Delta t}{2} A_{e i}\left(\varepsilon_{i, n}^{H}+\varepsilon_{i, n+1}^{H}\right)-\frac{\Delta t}{2} D_{e}\left(\varepsilon_{e, n}^{E}+\varepsilon_{e, n+1}^{E}\right) \\
& +\frac{\Delta t^{2}}{4} S_{e}\left[\left(-S_{e}^{T} \varepsilon_{e, n}^{E}+A_{i e}^{T} \varepsilon_{i, n}^{E}\right)-\left(S_{e}^{T} \varepsilon_{e, n+1}^{E}-A_{i e}^{T} \varepsilon_{i, n+1}^{E}\right)\right]+\Delta t \delta_{e, n}^{E}, \\
\varepsilon_{i, n+1}^{E}= & \varepsilon_{i, n}^{E}+\frac{\Delta t}{2} S_{i}\left(\varepsilon_{i, n}^{H}+\varepsilon_{i, n+1}^{H}\right)-\frac{\Delta t}{2} A_{i e}\left(\varepsilon_{e, n}^{H}+\varepsilon_{e, n+1}^{H}\right)-\frac{\Delta t}{2} D_{i}\left(\varepsilon_{i, n}^{E}+\varepsilon_{i, n+1}^{E}\right) \\
& -\frac{\Delta t^{2}}{4} A_{i e}\left[\left(-S_{e}^{T} \varepsilon_{e, n}^{E}+A_{i e}^{T} \varepsilon_{i, n}^{E}\right)-\left(S_{e}^{T} \varepsilon_{e, n+1}^{E}-A_{i e}^{T} \varepsilon_{i, n+1}^{E}\right)\right]+\Delta t \delta_{i, n}^{E}, \\
\varepsilon_{e, n+1}^{H}= & \varepsilon_{e, n}^{H}-\frac{\Delta t}{2} S_{e}^{T}\left(\varepsilon_{e, n}^{E}+\varepsilon_{e, n+1}^{E}\right)+\frac{\Delta t}{2} A_{i e}^{T}\left(\varepsilon_{i, n}^{E}+\varepsilon_{i, n+1}^{E}\right)+\Delta t \delta_{e, n}^{H}, \\
\varepsilon_{i, n+1}^{H}= & \varepsilon_{i, n}^{H}-\frac{\Delta t}{2} S_{i}^{T}\left(\varepsilon_{i, n}^{E}+\varepsilon_{i, n+1}^{E}\right)+\frac{\Delta t}{2} A_{e i}^{T}\left(\varepsilon_{e, n}^{E}+\varepsilon_{e, n+1}^{E}\right) \\
& +\frac{\Delta t^{2}}{4} A_{e i}^{T} A_{e i}\left(\varepsilon_{i, n+1}^{H}-\varepsilon_{i, n}^{H}\right)+\frac{\Delta t^{2}}{4} A_{e i}^{T} D_{e}\left(\varepsilon_{e, n+1}^{E}-\varepsilon_{e, n}^{E}\right)+\Delta t \delta_{i, n}^{H} .
\end{aligned}
$$

Let

$$
\varepsilon_{n}=\left(\begin{array}{c}
\varepsilon_{e, n}^{E} \\
\varepsilon_{i, n}^{E} \\
\varepsilon_{e, n}^{H} \\
\varepsilon_{i, n}^{H}
\end{array}\right) \quad \text { and } \quad \delta_{n}=\left(\begin{array}{c}
\delta_{e, n}^{E} \\
\delta_{i, n}^{E} \\
\delta_{e, n}^{H} \\
\delta_{i, n}^{H}
\end{array}\right),
$$

then from (3.27) we can write the global error in a more compact form (one-step recurrence relation)

$$
\varepsilon_{n+1}=R \varepsilon_{n}+\Delta t \rho_{n}, \quad R=R_{L}^{-1} R_{R}, \quad \rho_{n}=R_{L}^{-1} \delta_{n},
$$


where

$$
R_{L}=\left(\begin{array}{cccc}
I+\frac{\Delta t}{2} D_{e}-\frac{\Delta t^{2}}{4} S_{e} S_{e}^{T} & \frac{\Delta t^{2}}{4} S_{e} A_{i e}^{T} & -\frac{\Delta t}{2} S_{e} & \frac{\Delta t}{2} A_{e i} \\
\frac{\Delta t^{2}}{4} A_{i e} S_{e}^{T} & I+\frac{\Delta t}{2} D_{i}-\frac{\Delta t^{2}}{4} A_{i e} A_{i e}^{T} & \frac{\Delta t}{2} A_{i e} & -\frac{\Delta t}{2} S_{i} \\
\frac{\Delta t}{2} S_{e}^{T} & -\frac{\Delta t}{2} A_{i e}^{T} & I & 0 \\
-\frac{\Delta t}{2} A_{e i}^{T}-\frac{\Delta t^{2}}{4} A_{e i}^{T} D_{e} & \frac{\Delta t}{2} S_{i}^{T} & 0 & I-\frac{\Delta t^{2}}{4} A_{e i}^{T} A_{e i}
\end{array}\right),
$$

and $\varepsilon_{n}, \Delta t \rho_{n}$ and $\delta_{n}$ are respectively the (space-time) global, local and truncation errors.

Note that the recursion (3.29) has the standard form (see v.g. [16]) for the convergence analysis of one-step integration methods. It transfers local errors to the global error, essentially by adding all local errors. Indeed, for a given time interval $[0, T]$ we make the usual stability hypothesis

$$
\left\|R^{n}\right\| \leq K \quad \text { for } h \rightarrow 0 \text { and } n \geq 0, n \Delta t \leq T .
$$

On the other hand the elaboration of the error recursion (3.29) gives

$$
\varepsilon_{n}=R^{n} \varepsilon_{0}+R^{n-1} \Delta t \rho_{0}+\ldots+R \Delta t \rho_{n-2}+\Delta t \rho_{n-1},
$$

which leads directly (with (3.32)) to

$$
\left\|\varepsilon_{n}\right\| \leq K\left\|\varepsilon_{0}\right\|+K \Delta t \sum_{j=0}^{n-1}\left\|\rho_{j}\right\| \quad \text { for } n \Delta t \leq T .
$$

Recall that $\rho_{j}=R_{L}^{-1} \delta_{j}$ and because we assume stability we may consider $R_{L}$ inversely bounded for $\Delta t \sim h$, $h \rightarrow 0$. Consequently if $\delta_{j}=\mathcal{O}\left(\Delta t^{k}\right)$ we have $\left\|\rho_{j}\right\| \leq C \Delta t^{k}$. Assuming $\varepsilon_{0}=0$, we deduce from (3.34) that

$$
\left\|\varepsilon_{n}\right\| \leq \tilde{K} \Delta t^{k} \quad \text { for } n \Delta t \leq T
$$

with constant $\tilde{K}=K T C$.

\subsubsection{Error analysis}

We assume that the true PDE solutions $E_{e}^{h}, E_{i}^{h}, H_{e}^{h}$ and $H_{i}^{h}$ are sufficiently differentiable. Then we can Taylor expand the trapezoidal rule defect (3.26) at the symmetry point $t_{n+1 / 2}$ to get

$$
\delta_{E_{e}^{h}}\left(t_{n}\right)=\sum_{j=2^{\prime}} \frac{-j}{2^{j}(j+1) !}(\Delta t)^{j} E_{e}^{h(j+1)},
$$

where $j=2^{\prime}$ means even values for $j$ only and $E_{e}^{h(j)}$ denotes the $j$-th derivative of $E_{e}^{h}(t)$ at time $t=t_{n+1 / 2}$. We obtain similar expressions for $\delta_{E_{i}^{h}}\left(t_{n}\right), \delta_{H_{e}^{h}}\left(t_{n}\right)$ and $\delta_{H_{i}^{h}}\left(t_{n}\right)$. Note that these defects start with $\Delta t^{2}$ and 
the third solution derivative of the true PDE solution (in the first section we have assumed that the true PDE solution is three times differentiable). We conclude that the trapezoidal rule defects are $\mathcal{O}\left(\Delta t^{2}\right)$ for $\Delta t \sim h$ and $h \rightarrow 0$. Then, from (3.25), we write the truncation error as follows

$$
\delta_{n}=b_{n}+\mathcal{O}\left(\Delta t^{2}\right)
$$

where

$$
b_{n}=\left(\begin{array}{c}
b_{e, n}^{E} \\
b_{i, n}^{E} \\
b_{e, n}^{H} \\
b_{i, n}^{H}
\end{array}\right)=\left(\begin{array}{l}
\frac{\Delta t}{4} S_{e} \frac{\mathrm{d}}{\mathrm{d} t}\left(H_{e}^{h}\left(t_{n+1}\right)-H_{e}^{h}\left(t_{n}\right)\right) \\
-\frac{\Delta t}{4} A_{i e} \frac{\mathrm{d}}{\mathrm{d} t}\left(H_{e}^{h}\left(t_{n+1}\right)-H_{e}^{h}\left(t_{n}\right)\right) \\
0 \\
-\frac{\Delta t}{4} A_{e i}^{T} S_{e}\left(H_{e}^{h}\left(t_{n+1}\right)-H_{e}^{h}\left(t_{n}\right)\right)+\frac{\Delta t}{4} A_{e i}^{T} \frac{\mathrm{d}}{\mathrm{d} t}\left(E_{e}^{h}\left(t_{n+1}\right)-E_{e}^{h}\left(t_{n}\right)\right)
\end{array}\right)
$$

and $\mathcal{O}\left(\Delta t^{2}\right)$ contains the trapezoidal rule defects. Note that we have voluntarily omitted the spatial error parts contained in the $\sigma_{e}^{E}, \sigma_{i}^{E}, \sigma_{e}^{H}, \sigma_{i}^{H}$ contributions from (3.25) because our interest lies in temporal convergence order. Further carrying these spatial error contributions only complicates the formulas and will not lead to different conclusions for the temporal errors.

Next we Taylor expand the components of $b_{n}$ at the symmetry point $t_{n+1 / 2}$

$$
\begin{aligned}
b_{e, n}^{E} & =\frac{\Delta t}{4} S_{e} \sum_{j=1^{\prime}} \frac{1}{2^{j-1} j !}(\Delta t)^{j} H_{e}^{h(j+1)}, \\
b_{i, n}^{E} & =-\frac{\Delta t}{4} A_{i e} \sum_{j=1^{\prime}} \frac{1}{2^{j-1} j !}(\Delta t)^{j} H_{e}^{h(j+1)}, \\
b_{i, n}^{H} & =-\frac{\Delta t}{4} A_{e i}^{T} S_{e} \sum_{j=1^{\prime}} \frac{1}{2^{j-1} j !}(\Delta t)^{j} H_{e}^{h(j)}+\frac{\Delta t}{4} A_{e i}^{T} \sum_{j=1^{\prime}} \frac{1}{2^{j-1} j !}(\Delta t)^{j} E_{e}^{h(j+1)},
\end{aligned}
$$

where $j=1^{\prime}$ means odd values for $j$ only. For a fixed spatial dimension we find the expected second-order ODE convergence, since $S_{e}, A_{i e}$ and $A_{e i}^{T}$ are bounded for fixed dimension. Indeed, with (3.39) we have $b_{n}=\mathcal{O}\left(\Delta t^{2}\right)$ and because we assume stability we may consider $R_{L}$ inversely bounded, consequently $\rho_{n}=R_{L}^{-1} \delta_{n}=\mathcal{O}\left(\Delta t^{2}\right)$ and we conclude that we have the second-order convergence for a fixed dimension.

Now we observe, with (3.15), that under stable simultaneous space-time refinement, $\Delta t \sim h$ and $h \rightarrow 0$, we might lose one unit of $\Delta t$ in $b_{e, n}^{E}$ and $b_{i, n}^{E}$ (due to $S_{e}$ and $A_{i e}$, respectively) and two units of $\Delta t$ in $b_{i, n}^{H}$ (due to the product $A_{e i}^{T} S_{e}$ in the first terms). Then $b_{n}=\mathcal{O}(1)$, for $\Delta t \sim h$ and $h \rightarrow 0$ and with (3.35) we should expect a severe order reduction. However, as mentioned in [27], this result is based on standard local error analysis and in the transition from local to global errors it can happen that the order reduction for local errors is (partly) canceled. Often this cancellation can be shown to exist through a transformation of the global error recurrence to one by which we may gain one unit of $\Delta t$ in the transformed local error.

\subsubsection{A transformed global error recursion}

The transformation used in [27] emanates from [16], Lemma II.2.3. We write the latter for our one-step global error recursion (3.29) and stability assumption (3.32)

Lemma 3.1. Suppose the local error $\Delta \rho_{n}$ can be written as

$$
\Delta t \rho_{n}=(I-R) \xi_{n}+\eta_{n}
$$


with $\left\|\xi_{n}\right\| \leq C \Delta t^{k},\left\|\eta_{n}\right\| \leq C \Delta t^{k+1}$ and $\left\|\xi_{n+1}-\xi_{n}\right\| \leq C \Delta t^{k+1}$ for all $n$. Then there is a constant $C^{\prime}>0$, depending on $C, K$ and $T$, such that $\left\|\varepsilon_{n}\right\| \leq C^{\prime} \Delta t^{k}$ for $n \Delta t \leq T$.

The proof can be found in [16].

First we assume for $\Delta t \sim h, h \rightarrow 0$ that

$$
A_{e i}^{T} S_{e} H_{e}^{h(1)}=\mathcal{O}\left(\frac{1}{\Delta t}\right) .
$$

Consequently we get $b_{n}=\mathcal{O}(\Delta t)$ (in this case $b_{i, n}^{H}=\mathcal{O}(\Delta t)$, see (3.39)). With the above-mentioned Lemma we can assume that if the local error $\Delta t \rho_{n}$ allows a decomposition

$$
\Delta t \rho_{n}=(I-R) \xi_{n}+\eta_{n}
$$

such that $\xi_{n}=\mathcal{O}\left(\Delta t^{2}\right), \eta_{n}=\mathcal{O}\left(\Delta t^{3}\right)$ for $\Delta t \sim h, h \rightarrow 0$, then we have the desired second-order convergence for $\varepsilon_{n}$. So we need to verify (3.41), or equivalently,

$$
\Delta t \delta_{n}=\left(R_{L}-R_{R}\right) \xi_{n}+R_{L} \eta_{n},
$$

such that $\xi_{n}=\mathcal{O}\left(\Delta t^{2}\right), \eta_{n}=\mathcal{O}\left(\Delta t^{3}\right)$ for $\Delta t \sim h, h \rightarrow 0$.

Now we deal with the condition $\eta_{n}=\mathcal{O}\left(\Delta t^{3}\right)$. Recall that $\delta_{n}=b_{n}+\mathcal{O}\left(\Delta t^{2}\right)$, then (3.42) can be written as

$$
\Delta t\left(b_{n}+\mathcal{O}\left(\Delta t^{2}\right)\right)=\left(R_{L}-R_{R}\right) \xi_{n}+R_{L} \eta_{n} .
$$

Furthermore, $R_{L}$ is inversely bounded, then by assigning the $\mathcal{O}\left(\Delta t^{2}\right)$ terms present in (3.43) multiplied by $\Delta t R_{L}^{-1}$ for $\eta_{n}$ (i.e. $\eta_{n}=\Delta t R_{L}^{-1} \mathcal{O}\left(\Delta t^{2}\right)$ ), we have $\eta_{n}=\mathcal{O}\left(\Delta t^{3}\right)$. Consequently, we deduce from Lemma 3.1 that we have the desired second-order convergence if a vector $\xi_{n}$ exists such that $\xi_{n}=\mathcal{O}\left(\Delta t^{2}\right)$ for $\Delta t \sim h$, $h \rightarrow 0$ and

$$
\begin{gathered}
\Delta t b_{n}=\left(R_{L}-R_{R}\right) \xi_{n}, \text { i.e. } \\
\left(\begin{array}{cccc}
D_{e} & 0 & -S_{e} & A_{e i} \\
0 & D_{i} & A_{i e} & -S_{i} \\
S_{e}^{T} & -A_{i e}^{T} & 0 & 0 \\
-A_{e i}^{T} & S_{i}^{T} & 0 & 0
\end{array}\right)\left(\begin{array}{c}
\xi_{e, n}^{E} \\
\xi_{i, n}^{E} \\
\xi_{e, n}^{H} \\
\xi_{i, n}^{H}
\end{array}\right)=\left(\begin{array}{c}
b_{e, n}^{E} \\
b_{i, n}^{E} \\
b_{e, n}^{H} \\
b_{i, n}^{H}
\end{array}\right) .
\end{gathered}
$$

Equivalently, we have second-order convergence if a vector $\xi_{n}=\left[\left(\xi_{n}^{E}\right)^{T},\left(\xi_{n}^{H}\right)^{T}\right]^{T}$ exists such that $\xi_{n}=\mathcal{O}\left(\Delta t^{2}\right)$ for $\Delta t \sim h, h \rightarrow 0$ and

$$
\begin{aligned}
D \xi_{n}^{E}-S \xi_{n}^{H} & =b_{n}^{E}, \\
S^{T} \xi_{n}^{E} & =b_{n}^{H},
\end{aligned}
$$

where $\xi_{n}^{E}=\left[\left(\xi_{e, n}^{E}\right)^{T},\left(\xi_{i, n}^{E}\right)^{T}\right]^{T}, \xi_{n}^{H}=\left[\left(\xi_{e, n}^{H}\right)^{T},\left(\xi_{i, n}^{H}\right)^{T}\right]^{T}, b_{n}^{E}=\left[\left(b_{e, n}^{E}\right)^{T},\left(b_{i, n}^{E}\right)^{T}\right]^{T}, b_{n}^{H}=\left[\left(b_{e, n}^{H}\right)^{T},\left(b_{i, n}^{H}\right)^{T}\right]^{T}$.

Now we will check the existence of a such vector $\xi_{n}$. At this stage of the derivation we must be careful because the matrix $S$ is not necessarily a square matrix (in $2 \mathrm{D}$ this is not the case) and consequently $S$ may no be invertible. More precisely, if we denote $n_{d o f}$ the number of degrees of freedom, and if we consider the two-dimensional transversal magnetic (TM) model, then the size of the matrix $S$ is $n_{\text {dof }} \times 2 n_{\text {dof }}$. That is why we now use the notion of pseudo inverse. 
Definition 3.2. Let $A \in \mathbb{R}^{m \times n}, b \in \mathbb{R}^{m}, x \in \mathbb{R}^{n}$ and $A^{+}$the Moore-Penrose pseudo inverse of $A$ which is a generalization of the inverse and exists for any $m \times n$ matrix. If $A$ has full rank, then

$$
\begin{array}{ll}
A^{+}=A^{-1} & (m=n) \\
A^{+}=A^{T}\left(A A^{T}\right)^{-1} & (m<n), \\
A^{+}=\left(A^{T} A\right)^{-1} A^{T} & (m>n),
\end{array}
$$

and the solution of $A x=b$ is $x=A^{+} b$.

Assume the size of the matrix $S$ is $m \times n$ with $m \leq n$ (the case $m>n$ can be treated similarly). With the second equation of (3.46) and the above definition we derive

$$
\xi_{n}^{E}=\left(S^{T}\right)^{+} b_{n}^{H}
$$

With (2.10) we have

$$
\left(S^{T}\right)^{+}=\left(S S^{T}\right)^{-1} S \sim h, \quad \text { for } h \rightarrow 0,
$$

and recall that $b_{e, n}^{H}=0$ and $b_{i, n}^{H}=\mathcal{O}(\Delta t)$ (due to the initial assumption (3.40)) for $\Delta t \sim h, h \rightarrow 0$. Then we conclude with (3.48) and (3.49) that for $\Delta t \sim h, h \rightarrow 0$

$$
\xi_{n}^{E}=\mathcal{O}\left(\Delta t^{2}\right) .
$$

From the first equation of (3.46) we get

$$
\xi_{n}^{H}=-S^{+}\left(b_{n}^{E}-D \xi_{n}^{E}\right),
$$

and with (2.10) we get

$$
S^{+}=S^{T}\left(S S^{T}\right)^{-1} \sim h, \quad \text { for } h \rightarrow 0 .
$$

Recalling that $b_{e, n}^{E}=\mathcal{O}(\Delta t), b_{i, n}^{E}=\mathcal{O}(\Delta t)$ and $\xi_{n}^{E}=\mathcal{O}\left(\Delta t^{2}\right)$, we conclude from (3.51) and (3.52) that for $\Delta t \sim h, h \rightarrow 0$

$$
\text { if } D=0 \text { (no conduction term }-\sigma E \text { ) or } D=\mathcal{O}\left(h^{k}\right) \text { with } k \geq-1 \text {, then } \xi_{n}^{H}=\mathcal{O}\left(\Delta t^{2}\right) \text {. }
$$

With (3.50) and (3.53) we conclude that $\xi_{n}=\left[\left(\xi_{n}^{E}\right)^{T},\left(\xi_{n}^{H}\right)^{T}\right]^{T}=\mathcal{O}\left(\Delta t^{2}\right)$ for $\Delta t \sim h, h \rightarrow 0$ and consequently through the Lemma 3.1 we have the second-order convergence uniformly in $h$ under the assumption (3.40). Note that if we do not assume (3.40) (i.e. $b_{n}=\mathcal{O}(1)$ ) a similar proof, based on the same Lemma, only guarantees the first-order convergence. We can now state the following theorem

Theorem 3.3. Let $f^{H}(t), f^{E}(t) \in C^{2}[0, T]$ and suppose a Lax-Richtmyer stable space-time grid refinement $\Delta t \sim h, h \rightarrow 0$. On $[0, T]$ the approximations $H_{e}^{n}, H_{i}^{n}, E_{e}^{n}$ and $E_{i}^{n}$ of method (3.8) then converge to $H_{e}^{h}(t)$, $H_{i}^{h}(t), E_{e}^{h}(t)$ and $E_{i}^{h}(t)$

(i) at least at first order;

(ii) at least at second order, if in addition $A_{\text {ei }}^{T} S_{e} H_{e}^{h(1)}(t)=\mathcal{O}\left(\Delta t^{-1}\right)$ for $h \rightarrow 0$.

To sum up, we can guarantee at least the first-order convergence of method (3.8). As might be feared, component splitting can be detrimental to the temporal convergence order (order reduction). We have also put forward a sufficient condition (3.40) on the the true solution of the PDE problem for second-order convergence. However it would have been better if this sufficient condition could be controlled through the source term, because in general the true solution is of course not (a priori) known. 


\section{NumericAl RESUlts}

In this section we will conduct numerical tests in two dimensions by considering a wave propagation problem for which an analytical solution is available (see Sect. 4.1). Section 4.2 is devoted to the numerical convergence analysis of the locally implicit method (3.8). First in the ODE sense (i.e. on a fixed mesh) to illustrate that the method retains indeed its second-order convergence in time. Then in the PDE sense (i.e. for a simultaneous stable space-time grid refinement) to illustrate the reduction by one in the temporal convergence order, proved theoretically in Section 3.4. In the latter case we will also considered the second-order method (3.1) to ensure that the reduction order is due to component splitting and not to the spatial discretization itself. Finally in Section 4.3 we will present an overview of the influence of the reduction order on the efficiency of the locally implicit method, when high order approximation polynomials are used within the DG method. For that purpose we will also considered the methods (3.1)-(3.8).

\subsection{Simulation setting}

We solve the two-dimensional (2D) transverse magnetic (TM) model for the components $E^{z}(x, y, t)$, $H^{x}(x, y, t)$ and $H^{y}(x, y, t)$

$$
\left\{\begin{array}{l}
\mu \frac{\partial H^{x}}{\partial t}=-\frac{\partial E^{z}}{\partial y} \\
\mu \frac{\partial H^{y}}{\partial t}=\frac{\partial E^{z}}{\partial x} \\
\epsilon \frac{\partial E^{z}}{\partial t}=\frac{\partial H^{y}}{\partial x}-\frac{\partial H^{x}}{\partial y}-\sigma E^{z}-J_{E}^{z} .
\end{array}\right.
$$

In the following, we set $\epsilon=\mu=1$ and $\sigma=0$. Equations (4.1) are space discretized using a DG method formulated on quadrangular and triangular meshes, see Figure 1. In the preliminary implementation of this DG method, the approximation of the electromagnetic field components within a quadrangle $c_{i}$ or a triangle $\tau_{i}$ relies on a nodal $\mathbb{Q}_{l}$ or $\mathbb{P}_{l}$ interpolation method, respectively. The a priori convergence analysis for this DGTD method based on a centered numerical flux and formulated on simplicial meshes shows that the convergence rate is $\mathcal{O}\left(h^{l}\right)$ for a $l$-th interpolation order [10]. A quadrangle $c_{i}$ is characterized by the discretization parameters $\left(\Delta x_{i}, \Delta y_{i}\right)$ and a triangle $\tau_{i}$ by its height $h_{i}$. The critical step size, denoted $\Delta t_{c}$, used in the numerical tests is given by

$$
\Delta t_{c}=\left\{\begin{array}{l}
\frac{C F L}{\sqrt{\frac{1}{\Delta x_{k}^{2}}+\frac{1}{\Delta y_{k}^{2}}}, \text { on quadrangular meshes, }} \\
C F L \times h_{k}^{\text {min }}, \text { on triangular meshes, }
\end{array}\right.
$$

where the parameters $\left(\Delta x_{k}, \Delta y_{k}\right)$ characterize the smallest quadrangle $c_{k}$ treated explicitly and $h_{k}^{\min }$ the smallest height of a triangle $\tau_{k}$ inside the region treated explicitly. The value of the $C F L$ number corresponds to the numerical stability, i.e. the limit beyond which we observe a growth of the discrete energy. Finally for the component splitting we choose to treat implicitly the elements inside the red regions (see Fig. 1).

We consider the propagation of an eigenmode in a unitary perfectly electrically conducting (PEC) cavity. In this problem there is no source term i.e. $J_{E}^{z}=0$ in (4.1) and the exact solution is given by

$$
\left\{\begin{array}{l}
H^{x}(x, y, t)=-\frac{k \pi}{\omega} \sin (l \pi x) \cos (k \pi y) \sin (\omega t), \\
H^{y}(x, y, t)=\frac{l \pi}{\omega} \cos (l \pi x) \sin (k \pi y) \sin (\omega t) \\
E^{z}(x, y, t)=\sin (l \pi x) \sin (k \pi y) \cos (\omega t)
\end{array}\right.
$$



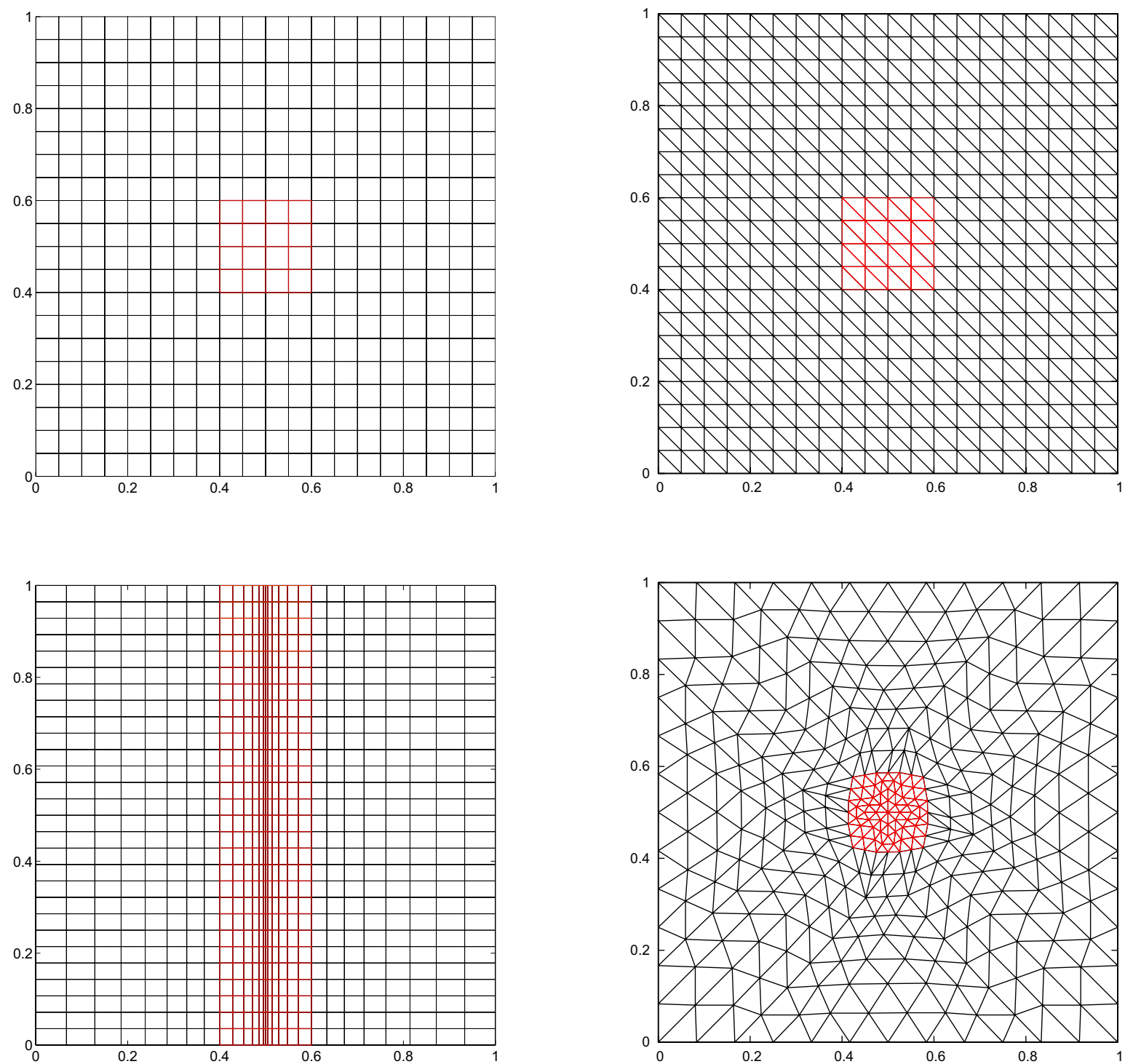

FiguRE 1. Examples of uniform/non-uniform, quadrangular/triangular meshes used in numerical tests.

where the resonance frequencies are given by

$$
\omega=\pi \sqrt{k^{2}+l^{2}}
$$

For numerical tests we put $k=l=1$ and we initialize the electromagnetic field with the exact analytical solution at $t=0$ i.e.

$$
\left\{\begin{array}{l}
H^{x}(x, y, t=0)=0, \\
H^{y}(x, y, t=0)=0, \\
E^{z}(x, y, t=0)=\sin (\pi x) \sin (\pi y) .
\end{array}\right.
$$




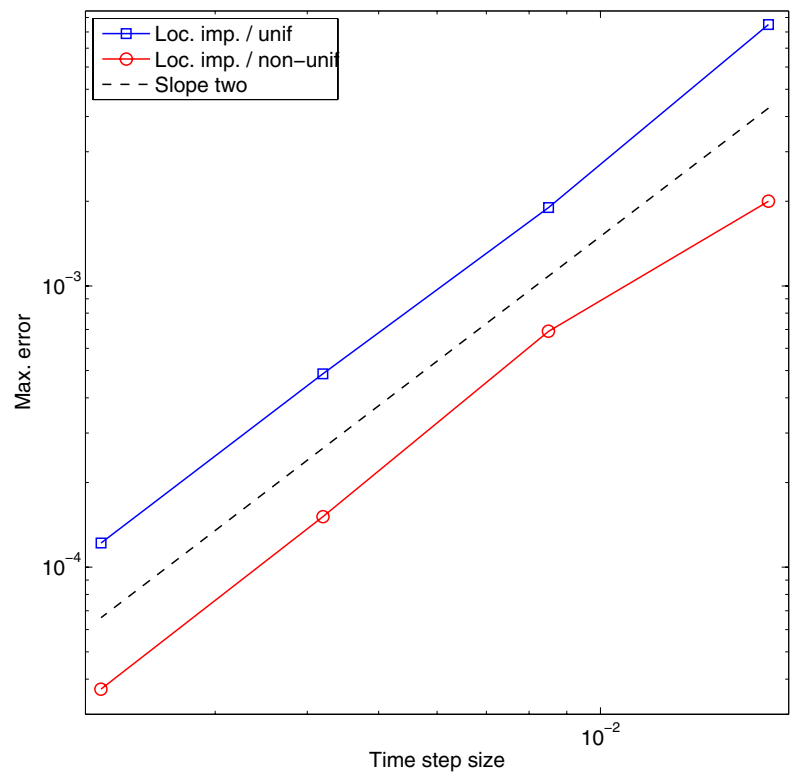

Figure 2. Temporal convergence (in the ODE sense) of the method (3.8) based on DGTD- $\mathbb{Q}_{2}$ method.

For the boundary conditions, we consider a PEC cavity such that the tangential component of the electric field vanishes on the boundaries

$$
n \times E^{z}=0 \text { on } \partial \Omega,
$$

where the domain $\Omega=[0,1]^{2}$ (the unitary PEC cavity) and $n$ denotes the unit outward normal to $\partial \Omega$.

\subsection{Numerical convergence analysis}

Throughout this subsection the total simulation time $T$ is set to $T=3.34 \times 10^{-9}$ s, and we use the DGTD- $\mathbb{Q}_{2}$ and DGTD- $\mathbb{P}_{2}$ methods so that the spatial error is not detrimental to the temporal convergence orders. For our integration method and the chosen spatial DG scheme the numerical $C F L$ number, used in (4.2), is equal to 0.24 and 0.20 for DGTD- $\mathbb{Q}_{2}$ and DGTD- $\mathbb{P}_{2}$ methods, respectively.

\subsubsection{Convergence in the $O D E$ sense}

To estimate the temporal convergence in the ODE sense we fix the discretization in space and we measure the electromagnetic field for different time steps (denotes by $W_{\Delta t}$ ) at the final time $T$. We plot the sup-norm of $W_{\Delta t / n}(T)-W_{\Delta t / 2 n}(T)$ as a function of $\Delta t / n$, in logarithmic scale. The use of the logarithmic scale allows to visualize the convergence rates as the slopes of the curves with o-marker and $\Delta$-marker for uniform and nonuniform meshes, respectively. The dashed line has slope two for second-order convergence. The results given in Figure 2 and Table 1 clearly confirm the expected behavior i.e. the second-order convergence of the method (3.8) for $\Delta t \rightarrow 0$ on a fixed space grid. 
TABLE 1. Temporal convergence (in the ODE sense), on uniform and non-uniform quadrangular meshes, of the method (3.8) based on the DGTD- $\mathbb{Q}_{2}$ method.

\begin{tabular}{lccc}
\hline$n$ & $\Delta t / n \rightarrow \Delta t / 2 n$ & \multicolumn{2}{c}{$\left\|W_{\Delta t / n}(T)-W_{\Delta t / 2 n}(T)\right\|_{\infty}$} \\
& & Uniform & Non-uniform \\
\hline 1 & $0.0169 \rightarrow 0.0085$ & $8.5000 \mathrm{e}-3$ & $2.0000 \mathrm{e}-3$ \\
2 & $0.0085 \rightarrow 0.0042$ & $1.9000 \mathrm{e}-3$ & $6.8912 \mathrm{e}-4$ \\
4 & $0.0042 \rightarrow 0.0021$ & $4.8669 \mathrm{e}-4$ & $1.5110 \mathrm{e}-4$ \\
8 & $0.0021 \rightarrow 0.0011$ & $1.2186 \mathrm{e}-4$ & $3.6888 \mathrm{e}-5$ \\
\hline Conv. rate & & 2.0249 & 1.9394 \\
\hline
\end{tabular}

\subsubsection{Convergence in the PDE sense}

First we conducted numerical investigations to check whether the condition (3.40) is satisfied. The following results have been obtained for $\Delta t \sim h, h \rightarrow 0$

$$
A_{e i}^{T} S_{e} H_{e}^{h(1)}=\left\{\begin{array}{l}
\mathcal{O}\left(\Delta t^{-1.3}\right), \text { for uniform meshes, } \\
\mathcal{O}\left(\Delta t^{-1.2}\right), \text { for non-uniform meshes. }
\end{array}\right.
$$

Consequently the condition (3.40) is not satisfied and we expect to observe a reduction PDE order by one.

The possible loss in accuracy could originate from the spatial discretization itself, regardless of component splitting. To eliminate this latter possibility we also repeat the numerical tests using the fully explicit method (3.1). Furthermore in [2] the authors have proven that DG methods on conformal tetrahedral/triangular meshes are spurious-free when the approximation spaces are made of elementwise polynomials of degree $l$ in each variable or local Nédélec elements of the first type of degree $l[21]$. They also remarked that this remains true for the local Nédélec elements of the first type of degree $l$ on conformal hexahedral/quadrilateral meshes but not for elementwise polynomials of degree $l$ in each variable or local Nédélec elements of the second type of degree $l[22]$. In the latter case spurious modes can appear. Similarly the methods (3.8)-(3.1) with a DG- $\mathbb{Q}_{2}$ spatial discretization on quadrangular meshes can produce spurious modes. Therefore, we have also conducted numerical tests on triangular meshes (see Fig. 1) with a DG- $\mathbb{P}_{2}$ spatial approximation to ensure that the reduction of convergence is not due to the emergence of spurious modes, but indeed to component splitting.

To estimate the temporal convergence in the PDE sense we measure the maximal $\mathrm{L}^{2}$-norm of the error for different meshes of increased resolution. We plot this error as a function of the square root of the number of degrees of freedom (DOF), in logarithmic scale. The obtained results, given in Figure 3 and Table 2, clearly confirm the theoretical behavior i.e. the first-order convergence of the method (3.8) and the second-order of (3.1), for $\Delta t \sim h, h \rightarrow 0$; except for (3.1) on non-uniform quadrangular meshes where we observe a super-convergence phenomenon (about 2.8 instead of 2.0 ).

\subsection{High order approximation within the DG method}

An attractive feature of the DG approach is the ability to easily increase the spatial convergence order. The reduction by one of the PDE order for the locally implicit method (3.8) raises the question of the efficiency of the method when high order approximation polynomials are used within the DG method. We have conducted a numerical investigation for the propagation of an eigenmode in a unitary PEC cavity (4.3). The total simulation time $T$ is set to $T=5 \times 10^{-8} \mathrm{~s}$ which corresponds to a propagation of the initial wave over 10 wavelengths. Note that the simulation time was chosen large enough in order that if we observe the evolution of the $\mathrm{L}^{2}$-norm error during time, the oscillations and the growth of the latter become very small from a time less than the final time. We plot the maximal $\mathrm{L}^{2}$-norm error as a function of the mesh spacing $h$ and the total number of DOF for the DGTD- $\mathbb{P}_{1,2,3,4}$ methods on uniform triangular meshes. The critical time step $\Delta t_{c}$ is determined by $(4.2)$ where the numerical $C F L$ number is given in Table 3. 

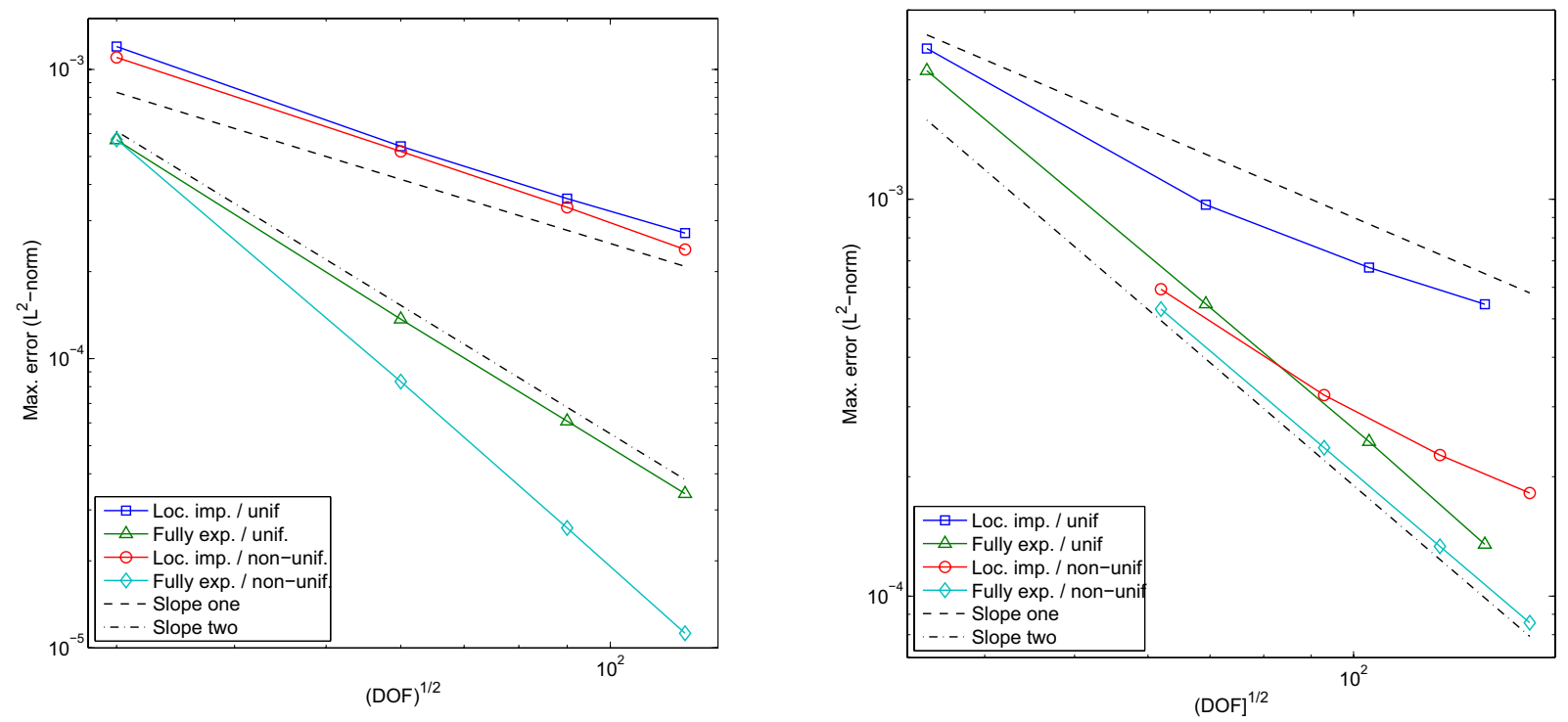

Figure 3. Temporal convergence (in the PDE sense), on uniform and non-uniform meshes, of methods (3.8) and (3.1) based on DGTD- $\mathbb{Q}_{2}$ (on left) and DGTD- $\mathbb{P}_{2}$ (on right) methods.

TABLE 2. Temporal convergence (in the PDE sense), on uniform and non-uniform quadrangular/triangular meshes (top/bottom), of methods (3.8) and (3.1) based on DGTD- $\mathbb{P}_{2}$ method.

\begin{tabular}{|c|c|c|c|c|c|c|c|}
\hline \multirow{2}{*}{$\begin{array}{c}\text { \# DOF } \\
\text { (unif. quadrangular } \\
\text { meshes) }\end{array}$} & \multicolumn{2}{|c|}{ Max. error in $\mathrm{L}^{2}$-norm } & \multirow{2}{*}{\multicolumn{2}{|c|}{$\begin{array}{c}\text { \# DOF } \\
\text { (non-unif. quadrangular } \\
\text { meshes) }\end{array}$}} & \multicolumn{3}{|c|}{ Max. error in $\mathrm{L}^{2}$-norm } \\
\hline & $\begin{array}{l}\text { Locally implicit } \\
\text { method }(3.8)\end{array}$ & $\begin{array}{l}\text { Fully explicit } \\
\text { method (3.1) }\end{array}$ & & & \multicolumn{2}{|c|}{$\begin{array}{l}\text { Locally implicit } \\
\text { method }(3.8)\end{array}$} & \multirow{2}{*}{$\begin{array}{l}\begin{array}{l}\text { Fully exp } \\
\text { method }\end{array} \\
5.7274\end{array}$} \\
\hline 900 & $1.2000 \mathrm{e}-3$ & $5.7020 \mathrm{e}-4$ & 900 & & \multicolumn{2}{|c|}{$1.1000 \mathrm{e}-3$} & \\
\hline 3600 & $5.4236 \mathrm{e}-4$ & $1.3695 \mathrm{e}-4$ & 3600 & & \multicolumn{2}{|c|}{$5.2066 \mathrm{e}-4$} & $8.3295 \mathrm{e}$ \\
\hline 8100 & $3.5759 \mathrm{e}-4$ & $6.0818 \mathrm{e}-5$ & 8100 & & \multicolumn{2}{|c|}{$3.3373 \mathrm{e}-4$} & $2.5979 \mathrm{e}$ \\
\hline 14400 & $2.7164 \mathrm{e}-4$ & $3.4116 \mathrm{e}-5$ & 14400 & & \multicolumn{2}{|c|}{$2.3845 \mathrm{e}-4$} & $1.1239 \mathrm{e}$ \\
\hline Conv. rate & 1.0762 & 2.0315 & & & \multicolumn{2}{|c|}{1.0991} & 2.83 \\
\hline \# DOF & \multicolumn{2}{|c|}{ Max. error in $L^{2}$-norm } & \# DOF & \multicolumn{4}{|c|}{ Max. error in $\mathrm{L}^{2}$-norm } \\
\hline $\begin{array}{l}\text { (unif. triangular } \\
\text { meshes) }\end{array}$ & $\begin{array}{cc}\text { Locally implicit } & \mathrm{F} x \\
\text { method }(3.8) & \mathrm{m}\end{array}$ & $\begin{array}{l}\text { Wully explicit } \\
\text { nethod (3.1) }\end{array}$ & $\begin{array}{l}\text { non-unif. triangular } \\
\text { meshes })\end{array}$ & \multicolumn{2}{|c|}{$\begin{array}{l}\text { Locally implicit } \\
\text { method }(3.8)\end{array}$} & \multicolumn{2}{|c|}{$\begin{array}{l}\text { Fully explicit } \\
\text { method (3.1) }\end{array}$} \\
\hline 1200 & $2.3990 \mathrm{e}-3$ & $2.1098 \mathrm{e}-3$ & 3840 & \multicolumn{2}{|c|}{$5.9380 \mathrm{e}-4$} & \multicolumn{2}{|c|}{$5.2875 \mathrm{e}-4$} \\
\hline 4800 & $9.6905 \mathrm{e}-4$ & $5.4487 \mathrm{e}-4$ & 8640 & \multicolumn{2}{|c|}{$3.2121 \mathrm{e}-4$} & \multicolumn{2}{|c|}{$2.3662 \mathrm{e}-4$} \\
\hline 10800 & $6.7305 \mathrm{e}-4$ & $2.4522 \mathrm{e}-4$ & 15360 & \multicolumn{2}{|c|}{$2.2685 \mathrm{e}-4$} & \multicolumn{2}{|c|}{$1.3354 \mathrm{e}-4$} \\
\hline 19200 & $5.4399 \mathrm{e}-4$ & $1.3502 \mathrm{e}-4$ & 24000 & \multicolumn{2}{|c|}{$1.8200 \mathrm{e}-4$} & \multicolumn{2}{|c|}{$8.5705 \mathrm{e}-5$} \\
\hline Conv. rate & 1.0817 & 1.9775 & & \multicolumn{2}{|c|}{1.2988} & \multicolumn{2}{|c|}{1.9859} \\
\hline
\end{tabular}

TABLE 3. Numerical value of the $C F L$ number in (4.2), for triangular meshes.

\begin{tabular}{ccccc}
\hline Method & DGTD- $\mathbb{P}_{4}$ & DGTD- $\mathbb{P}_{3}$ & DGTD- $\mathbb{P}_{2}$ & DGTD- $\mathbb{P}_{1}$ \\
\hline Numerical $C F L$ & 0.09 & 0.13 & 0.20 & 0.30 \\
\hline
\end{tabular}


TABLE 4. Rate of decrease of the error relatively to the polynomial degree, for methods (3.8)(3.1), for a fixed number of DOF to $10000\left(\operatorname{errorL}^{2}\left(\mathbb{P}_{l}\right)=\left[1-r\left(\mathbb{P}_{k}, \mathbb{P}_{l}\right)\right] \times \operatorname{errorL}^{2}\left(\mathbb{P}_{k}\right)\right)$.

\begin{tabular}{lcc}
\hline$r\left(\mathbb{P}_{k}, \mathbb{P}_{l}\right)$ & $\mid$ error $\mathrm{L}^{2}\left(\mathbb{P}_{k}\right)-$ error $\mathrm{L}^{2}\left(\mathbb{P}_{l}\right)|/| \operatorname{error} \mathrm{L}^{2}\left(\mathbb{P}_{k}\right) \mid$ \\
& Method $(3.8)$ & Method $(3.1)$ \\
\hline$r\left(\mathbb{P}_{2}, \mathbb{P}_{3}\right)$ & 0.14 & 0.34 \\
$r\left(\mathbb{P}_{2}, \mathbb{P}_{4}\right)$ & 0.28 & 0.52 \\
$r\left(\mathbb{P}_{3}, \mathbb{P}_{4}\right)$ & 0.17 & 0.28 \\
\hline
\end{tabular}

TABLE 5. Rate of decrease of the error relatively to the polynomial degree, for methods (3.8)-(3.1), for a fixed error tolerance to $10^{-3}\left(\operatorname{DOF}\left(\mathbb{P}_{l}\right)=\left[1-r\left(\mathbb{P}_{k}, \mathbb{P}_{l}\right)\right] \times \operatorname{DOF}\left(\mathbb{P}_{k}\right)\right)$.

\begin{tabular}{|c|c|c|}
\hline \multirow[t]{2}{*}{$r\left(\mathbb{P}_{k}, \mathbb{P}_{l}\right)$} & \multicolumn{2}{|c|}{$\left|D O F\left(\mathbb{P}_{k}\right)-D O F\left(\mathbb{P}_{l}\right)\right| /\left|D O F\left(\mathbb{P}_{k}\right)\right|$} \\
\hline & Method (3.8) & Method (3.1) \\
\hline$r\left(\mathbb{P}_{2}, \mathbb{P}_{3}\right)$ & 0.11 & 0.34 \\
\hline$r\left(\mathbb{P}_{2}, \mathbb{P}_{4}\right)$ & 0.33 & 0.52 \\
\hline$r\left(\mathbb{P}_{3}, \mathbb{P}_{4}\right)$ & 0.26 & 0.28 \\
\hline
\end{tabular}

We observe in Figure 4 that for a given error the locally implicit method needs a finer grid or significantly more DOF compared to the fully explicit case. In other words the method (3.8) needs more points per wavelength. We have also indicated in Tables 4,5 the rates of decrease of the error relatively to the polynomial degree for both methods, for a given error tolerance $\left(\mathrm{L}^{2}\right.$-norm error $\left.=10^{-3}\right)$ or a given total number of DOF $(=10000)$. As expected these rates are lower for (3.8) than for (3.1).

Because of its first order temporal convergence, an high order spatial discretization is less advantageous for method (3.8) than for the fully explicit method (3.1) which retains its second-order PDE convergence. Nevertheless increasing the polynomial order remains relatively useful for improving the accuracy as shown in Figure 4 and Tables 4,5. Furthermore, as previously mentioned in the introduction, another attractive feature of th DG approach is to handle geometrical details by using locally refined space grids. Consequently we can expect that the higher cost of the locally implicit method compared to the fully explicit method (in the sense of number of ppw for a given error) can be partly compensated because the unduly large step size restriction of (3.1) is overcome when a local refinement is necessary for a practical time dependent problem.

\section{Conclusion And Future WORK}

In this paper, we have shown that component splitting can be detrimental to the temporal convergence uniformaly in $h$ of a locally implicit DG method. We have presented a sufficient condition (see (3.40)) on the true PDE solution to recover the second-order convergence. Thus in the general case we can only guarantee first-order convergence. Numerical investigations have been conducted to illustrate this last theoretical result in $2 \mathrm{D}$ by considering a wave propagation problem for which an analytical solution is available. Note that the aim of these tests was not to illustrate the computational efficiency of the locally implicit DG method (for that purpose see [7]) but the temporal convergence order of the method (3.8) in the ODE and PDE senses (i.e. for $\Delta t \rightarrow 0$ on a fixed space grid and $\Delta t \sim h, h \rightarrow 0$, respectively).

In this paper we have mentioned another component splitting method from [27] which cover the common spatial discretizations like finite difference and various finite element discretizations. We plan to compare the latter with the implicit-explicit integration method from $[7,23]$ for a DG discretization on unstructured meshes. The objective will be to illustrate the practical virtue of both component splitting methods using locally refined space grids, and to highlight the most efficient one which then can be advocated for future use. 

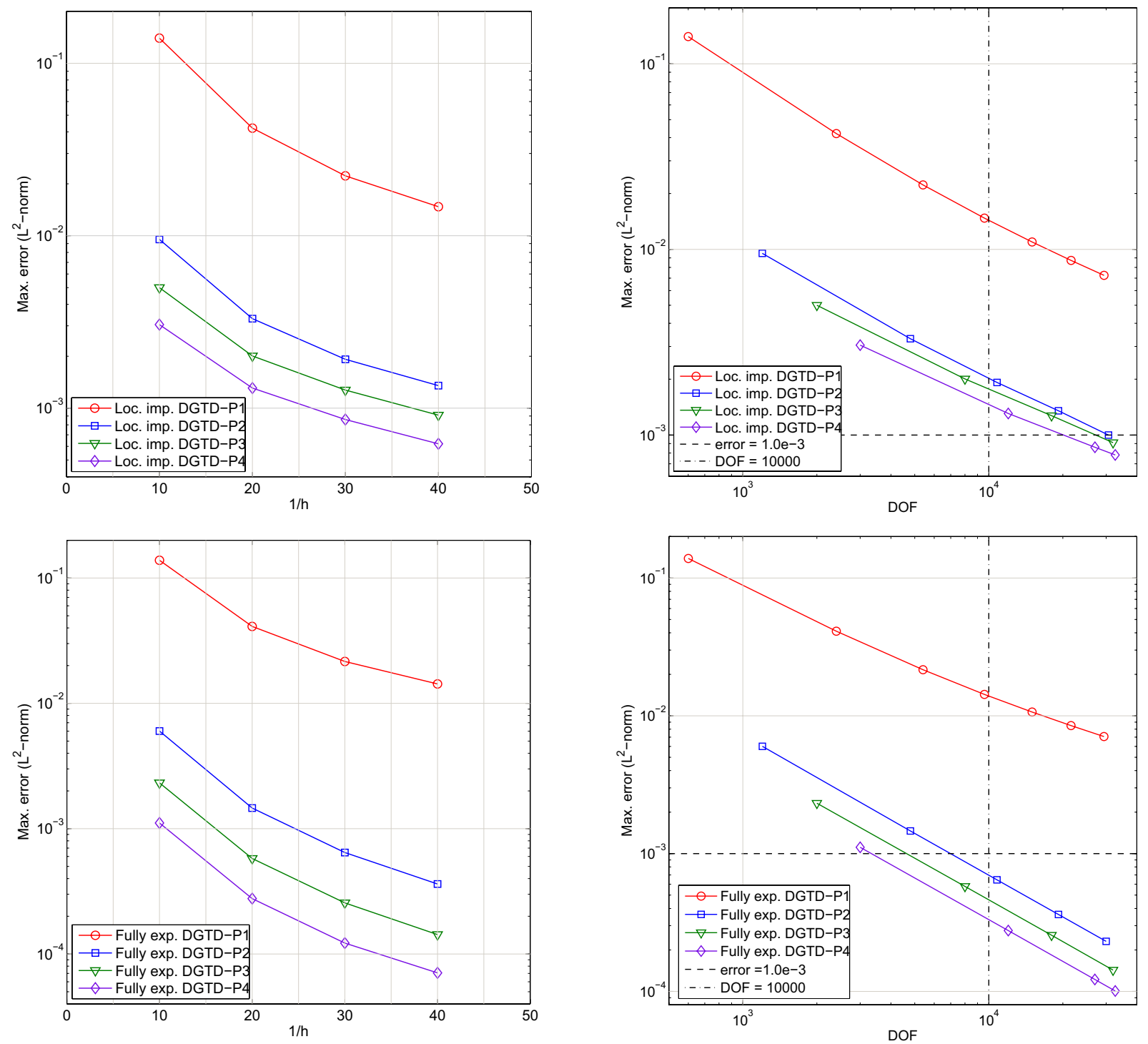

FIgURE 4. Maximal $\mathrm{L}^{2}$-norm error as function of the mesh spacing $h$ and the total number of DOF (left - right) for methods (3.8)-(3.1) (top - bottom) based on DGTD- $\mathbb{P}_{1,2,3,4}$ spatial discretization.

In Section 4.3 we have seen that the reduction by one in the PDE convergence order for the method (3.8) does not allow to fully exploit the gain in accuracy and efficiency that one can expect when the interpolation order is increased. Thus a future objective is to examine higher order implicit-explicit methods. Several strategies based on one of the schemes from [23,27] can be envisaged. One possibility is to exploit composition methods [13], known to be accurate with well-designed composition coefficients $[19,25,32]$ to minimize truncation errors. Note that these composition methods with orders beyond two are restricted to problems with small (non-stiff) dissipative terms [1]. Furthermore in the presence of source functions, the convergence order may be lower than the chosen composition order [28]. In the latter reference the author used the scheme (3.1) as the basis method in composition and a source function perturbation to solve Maxwell's equations. With this perturbed 
scheme the author obtained at least a third-order scheme, and a fourth-order one with additional sufficient conditions. Another possibility is to rely on local or global Richardson extrapolations $[1,9,12,18,30]$ which are easy to implement and straightforward to parallelize. These high order extensions are well-known in the numerical solution of ODEs and have been already considered with (3.1) as the basis methods to solve damped Maxwell equations [1]. With Dirichlet boundary conditions the authors of [1] observed that the global approach does not suffer from order reduction; this is not the case of composition methods and local extrapolations. However when the reduction order is inevitable, they advocate the local approach that allows to eliminate error terms instantaneously. The fact that these different methods, with an implicit-explicit approach, retain their ODE convergence order for simultaneous space-time grid refinement towards the true PDE solution is doubtful. Moreover, due to their accuracy, a comparison with high order local time stepping approaches, as in [11,26], will certainly be very interesting.

Acknowledgements. The author wishes to acknowledge the many and important contributions to this work by Prof. dr. Jan Verwer of Centrum Wiskunde en Informatica (CWI, 1090 GB Amsterdam, The Netherlands), who passed away on 16 February 2011 before completion of this paper. The author wishes also to thank the CWI for its hospitality during the month of December 2010, period during which much of the theory presented in this paper was conducted in collaboration with Jan Verwer. Stéphane Descombes and Stéphane Lanteri are acknowledged for valuable discussions and important assistance throughout this work.

\section{REFERENCES}

[1] M.A. Botchev and J.G. Verwer, Numerical integration of damped maxwell equations. SIAM J. Sci. Comput. 31 (2009) 13221346.

[2] A. Buffa and I. Perugia, Discontinuous Galerkin approximation of the Maxwell eigenproblem. SIAM J. Numer. Anal. 44 (2006) $2198-2226$.

[3] A. Catella, V. Dolean and S. Lanteri, An unconditionally stable discontinuous galerkin method for solving the 2-D time-domain Maxwell equations on unstructured triangular meshes. IEEE Trans. Magn. 44 (2008) 1250-1253.

[4] B. Cockburn, G.E.G.E. Karniadakis and C.-W. Shu Eds., Discontinuous Galerkin methods. Theory, computation and applications. Springer-Verlag, Berlin (2000)

[5] G. Cohen, X. Ferrieres and S. Pernet, A spatial high order hexahedral discontinuous Galerkin method to solve Maxwell's equations in time-domain. J. Comput. Phys. 217 (2006) 340-363.

[6] J. Diaz and M.J. Grote, Energy conserving explicit local time-stepping for second-order wave equations. SIAM J. Sci. Comput. 31 (2009) 1985-2014.

[7] V. Dolean, H. Fahs, L. Fezoui and S. Lanteri, Locally implicit discontinuous Galerkin method for time domain electromagnetics. J. Comput. Phys. 229 (2010) 512-526.

[8] H. Fahs, Development of a hp-like discontinuous Galerkin time-domain method on non-conforming simplicial meshes for electromagnetic wave propagation. Int. J. Numer. Anal. Mod. 6 (2009) 193-216.

[9] I. Faragó, Á. Havasi and Z. Zlatev, Richardson-extrapolated sequential splitting and its application. J. Comput. Appl. Math. 234 (2010) 3283-3302.

[10] L. Fezoui, S. Lanteri, S. Lohrengel and S. Piperno, Convergence and stability of a discontinuous Galerkin time-domain method for the 3D heterogeneous Maxwell equations on unstructured meshes. ESAIM: M2AN 39 (2005) 1149-1176.

[11] M.J. Grote and T. Mitkova, Explicit local time stepping methods for Maxwell's equations. J. Comput. Appl. Math. 234 (2010) 3283-3302.

[12] E. Hairer and G. Wanner, Solving Ordinary Differential Equations II - Stiff and Differential-Algebraic problems, 2nd edition. Springer-Verlag, Berlin (1996).

[13] E. Hairer, C. Lubich and G. Wanner, Geometric Numerical Integration, 2nd edition. Springer-Verlag, Berlin (2002).

[14] J. Hesthaven and T. Warburton, Nodal high-order methods on unstructured grids. I. Time-domain solution of Maxwell's equations. J. Comput. Phys. 181 (2002) 186-221.

[15] J. Hesthaven and T. Warburton, Nodal Discontinuous Galerkin Methods. Springer (2008).

[16] W. Hundsdorfer and J.G. Verwer, Numerical Solution of Time-Dependent Advection-Diffusion-Reaction Equations. SpringerVerlag, Berlin (2003).

[17] J. Jin, The Finite Element Method in Electromagnetics, 2nd edition. Wiley-IEEE Press (2002).

[18] G.Yu. Kulikov, Local theory of extrapolation methods. Numer. Algorithm 53 (2010) 321-342

[19] R.I. McLachlan, On the numerical integration of ordinary differential equations by symmetric composition methods. SIAM J. Sci. Comput. 16 (1995) 151-168.

[20] E. Montseny, S. Pernet, X. Ferrires and G. Cohen, Dissipative terms and local time-stepping improvements in a spatial high order Discontinuous Galerkin scheme for the time-domain Maxwell's equations. J. Comput. Phys. 227 (2008) 6795-6820. 
[21] J.C. Nédélec, Mixed finite elements in $\mathbb{R}^{3}$. Numer. Math. 35 (1980) 315-341.

[22] J.C. Nédélec, A new dfamily of mixed finite elements in $\mathbb{R}^{3}$. Numer. Math. 50 (1986) 57-81.

[23] S. Piperno, Symplectic local time-stepping in non-dissipative DGTD methods applied to wave propagation problem. ESAIM: M2AN 40 (2006) 815-841.

[24] M. Remaki, A new finite volume scheme for solving Maxwell's system. Compel 19 (2000) 913-931.

[25] M. Suzuki, Fractal decomposition of exponential operators with applications to many-body theories and Monte-Carlo simulations. Phys. Lett. A 146 (1990) 319-323.

[26] A. Taube, M. Dumbser, C.D. Munz and R. Schneider, A high order discontinuous Galerkin method with local time stepping for the Maxwell equations. Int. J. Numer. Model. 22 (2009) 77-103.

[27] J.G. Verwer, Component splitting for semi-discrete Maxwell equations. BIT Numer. Math. 51 (2011) $427-445$.

[28] J.G Verwer, Composition methods, Maxwell's and source term. CWI Technical report (2010); Available at http://oai.cwi.nl/oai/asset/17036/17036A.pdf .

[29] J.G. Verwer and M.A. Botchev, Unconditionaly stable integration of Maxwell's equations. Linear Algebra Appl. 431 (2009) $300-317$.

[30] J.G. Verwer and H.B. de Vries, Global extrapolation of a first order splitting method. SIAM J. Sci. Stat. Comput. 6 (1985) $771-780$.

[31] K.S. Yee, Numerical solution of initial boundary value problems involving Maxwell's equations in isotropic media. IEEE Trans. Antennas Propag. 14 (1966) 302-307.

[32] H. Yoshida, Construction of higher order symplectic integrators. Phys. Lett. A 150 (1990) 262-268. 